\title{
Characterizations of Ordered Semigroups by New Type of Interval Valued Fuzzy Quasi-Ideals
}

\author{
Jian Tang, ${ }^{1,2}$ Xiangyun Xie, ${ }^{3}$ and Yanfeng Luo ${ }^{1}$ \\ ${ }^{1}$ School of Mathematics and Statistics, Lanzhou University, Lanzhou, Gansu 730000, China \\ ${ }^{2}$ School of Mathematics and Finance, Fuyang Normal College, Fuyang, Anhui 236037, China \\ ${ }^{3}$ School of Mathematics and Computational Science, Wuyi University, Jiangmen, Guangdong 529020, China
}

Correspondence should be addressed to Jian Tang; tangj13@lzu.edu.cn

Received 17 February 2014; Accepted 7 May 2014; Published 25 May 2014

Academic Editor: Hak-Keung Lam

Copyright (C) 2014 Jian Tang et al. This is an open access article distributed under the Creative Commons Attribution License, which permits unrestricted use, distribution, and reproduction in any medium, provided the original work is properly cited.

\begin{abstract}
The concept of non- $k$-quasi-coincidence of an interval valued ordered fuzzy point with an interval valued fuzzy set is considered. In fact, this concept is a generalized concept of the non- $k$-quasi-coincidence of a fuzzy point with a fuzzy set. By using this new concept, we introduce the notion of interval valued $\left(\bar{\epsilon}, \bar{\epsilon} \vee \overline{q_{\bar{k}}}\right)$-fuzzy quasi-ideals of ordered semigroups and study their related properties. In addition, we also introduce the concepts of prime and completely semiprime interval valued $\left(\bar{\epsilon}, \bar{\epsilon} \vee \overline{q_{\bar{k}}}\right)$-fuzzy quasiideals of ordered semigroups and characterize bi-regular ordered semigroups in terms of completely semiprime interval valued $\left(\bar{\epsilon}, \bar{\epsilon} \vee \overline{q_{\bar{k}}}\right)$-fuzzy quasi-ideals. Furthermore, some new characterizations of regular and intra-regular ordered semigroups by the properties of interval valued $\left(\bar{\epsilon}, \bar{\epsilon} \vee \overline{q_{\bar{k}}}\right)$-fuzzy quasi-ideals are given.
\end{abstract}

\section{Introduction}

The theory of fuzzy sets was first developed by Zadeh [1] and has been applied to many branches in mathematics. Rosenfeld [2] inspired the fuzzification of algebraic structures and introduced the notion of fuzzy subgroups. Later on, Zadeh [3] also introduced the concept of interval valued fuzzy set by considering the values of the membership functions as the intervals of numbers instead of the numbers alone. An interval valued fuzzy set is a generalized form of the ordinary fuzzy sets and provides a more adequate description of uncertainty than the ordinary fuzzy sets. The interval valued fuzzy subgroups were first defined and studied by Biswas [4] which are the subgroups of the same nature of the fuzzy subgroups defined by Rosenfeld. Interval valued fuzzy subsets have been applied extensively in different kinds of fields of mathematics (see [5-10]). A new kind of fuzzy subgroup, that is, the $(\epsilon, \in \vee q)$-fuzzy subgroup, was then introduced by Bhakat and Das $[11,12]$ by using the combined notions of "belongingness" and "quasicoincidence" of fuzzy points and fuzzy sets proposed by $\mathrm{Pu}$ and Liu in [13]. In fact, the $(\epsilon, \in \vee q)$-fuzzy subgroup is an important and useful generalization of Rosenfeld's fuzzy subgroup. Since then, many researchers used the idea of generalized fuzzy sets and gave several results in different branches of algebra. For more details, the reader is referred to [14-20].

In mathematics, an ordered semigroup is a semigroup together with a partial order that is compatible with the semigroup operation. Ordered semigroups have several applications in the theory of sequential machines, formal languages, computer arithmetics, and error-correcting codes. In [21], Kehayopulu and Tsingelis applied the concept of fuzzy sets to the theory of ordered semigroups. Then they defined "fuzzy" analogous of several notations, which appeared to be useful in the theory of ordered semigroups. The theory of fuzzy sets on ordered semigroups has been recently developed (see [22-30]). Recently, Khan et al. [31] introduced the concept of interval valued $(\alpha, \beta)$-fuzzy biideals of ordered semigroups by using the idea of a quasicoincidence of an interval valued fuzzy point with an interval valued fuzzy set. In particular, they studied the properties of interval valued $(\epsilon, \in \vee q)$-fuzzy biideals in detail. Furthermore, Davvaz et al. [32] introduced the concept of interval valued generalized fuzzy filters of an 
ordered semigroup and obtained many interesting related results.

As we know, quasi-ideals play an important role in the study of ring, semigroup, and ordered semigroup structures. The concept of a quasi-ideal in rings and semigroups was studied by Stienfeld in [33]. Furthermore, Kehayopulu and Tsingelis extended the concept of quasi-ideals in ordered semigroups as a nonempty subset $Q$ of an ordered semigroup $S$ such that (1) $(Q S] \cap(S Q] \subseteq Q$ and (2) if $a \in Q$ and $S \ni b \leq a$, then $b \in Q$ (see [34]). The fuzzy quasi-ideals in ordered semigroups were studied in $[23,27]$, where the basic properties of ordered semigroups in terms of fuzzy quasiideals are given. Motivated by the study of fuzzy quasi-ideals in rings, semigroups and ordered semigroups, and also motivated by Davvaz and Khan's works in ordered semigroups in terms of interval valued fuzzy subsets, we attempt in the present paper to study a new type of interval valued fuzzy quasi-ideals in an ordered semigroup in detail. The rest of this paper is organized as follows. In Section 2, we recall some basic definitions and results of ordered semigroups which will be used throughout this paper. In Section 3, we introduce the concept of interval valued $\left(\bar{\epsilon}, \bar{\epsilon} \vee \overline{q_{\tilde{k}}}\right)$-fuzzy quasi-ideals of an ordered semigroup $S$ by the interval valued ordered fuzzy points of $S$ and investigate some related properties. In particular, we discuss the relationships interval valued fuzzy quasi-ideals and interval valued $\left(\bar{\epsilon}, \bar{\epsilon} \vee \overline{q_{\tilde{k}}}\right)$-fuzzy quasiideals of an ordered semigroup. The idea of prime interval valued $\left(\bar{\epsilon}, \bar{\epsilon} \vee \overline{q_{\tilde{k}}}\right)$-fuzzy quasi-ideals in ordered semigroups is given and the related theorems are provided in Section 4. In Section 5, we first give some characterizations of regular and intra-regular ordered semigroups by the properties of interval valued $\left(\bar{\epsilon}, \bar{\epsilon} \vee \overline{q_{\tilde{k}}}\right)$-fuzzy quasi-ideals. Furthermore, we introduce the notion of completely semiprime interval valued $\left(\bar{\epsilon}, \bar{\epsilon} \vee \overline{q_{\tilde{k}}}\right)$-fuzzy quasi-ideals of ordered semigroups and characterize biregular ordered semigroups in terms of completely semiprime interval valued $\left(\bar{\epsilon}, \bar{\epsilon} \vee \overline{q_{\widetilde{k}}}\right)$-fuzzy quasiideals. Some conclusions are given in the last Section.

\section{Preliminaries and Some Notations}

Recall that an ordered semigroup $(S, \cdot, \leq)$ is a semigroup $(S, \cdot)$ with an order relation " $\leq$ " such that $a \leq b$ implies $x a \leq x b$ and $a x \leq b x$ for any $x \in S$.

Let $S$ be an ordered semigroup. For $H \subseteq S$, we define

$$
(H]:=\{t \in S \mid t \leq h \text { for some } h \in H\} .
$$

For $H=\{a\}$, we write $(a]$ instead of $(\{a\}]$. For two subsets $A$, $B$ of $S$, we have (1) $A \subseteq(A]$; (2) if $A \subseteq B$, then $(A] \subseteq(B]$; (3) $(A](B] \subseteq(A B]$; (4) $((A]]=(A] ;(5))((A])(B])=(A B]$ (see [35]).

By a subsemigroup of $S$ we mean a nonempty subset $A$ of $S$ such that $A^{2} \subseteq A$. A quasi-ideal $Q$ of an ordered semigroup $S$ is called prime if for any two elements $x, y$ of $S$, such that $x y \in$ $\mathrm{Q}$, then $x \in \mathrm{Q}$ or $y \in \mathrm{Q}$. We denote by $\mathrm{Q}(a)$ the quasi-ideal of $S$ generated by $a(a \in S)$. Then $Q(a)=(a \cup((a S] \cap(S a])]$.

An ordered semigroup $(S, \cdot, \leq)$ is called regular if, for each $a \in S$, there exists $x \in S$ such that $a \leq a x a$. Equivalent definitions: (1) $A \subseteq$ (ASA], $\forall A \subseteq S$. (2) $a \in(a S a], \forall a \in S$ (see [34]). An ordered semigroup $(S, \cdot, \leq)$ is called intraregular if, for each $a \in S$, there exist $x, y \in S$ such that $a \leq x a^{2} y$. Equivalent definitions: (1) $A \subseteq\left(S A^{2} S\right], \forall A \subseteq$ S. (2) $a \in\left(S a^{2} S\right], \forall a \in S$ (see [34]).

We next state some fuzzy logic concepts. Recall that a fuzzy subset $f$ of an ordered semigroup $S$ is a function from $S$ into the real closed interval $[0,1]$, that is, $f: S \rightarrow[0,1]$. A fuzzy subset $f$ of an ordered semigroup $S$ is called a fuzzy quasi-ideal of $S$ if (1) $x \leq y \Rightarrow f(x) \geq f(y)$ for all $x, y \in S$, and $(2)(f \circ S) \cap(S \circ f) \subseteq f($ see $[23])$.

By an interval number $\tilde{a}$ we mean an interval $\left[a^{-}, a^{+}\right]$, where $0 \leq a^{-} \leq a^{+} \leq 1$. The set of all interval numbers is denoted by $D[0,1]$. The interval number $[a, a]$ can be simply identified by the number $a \in[0,1]$. In particular, we define the set $E D[\alpha, \beta]:=\{\widetilde{a} \in D[0,1] \mid \tilde{a}=[a, a], \alpha \leq a \leq \beta\}$, where $0 \leq \alpha \leq \beta \leq 1$. For the interval numbers $\tilde{a}_{j}=\left[a_{j}^{-}, a_{j}^{+}\right]$, $j \in I$, we define the operations " $\wedge$ ", " $\vee$ ", “ $\leq$ ", “=”, “<”, “+” and "_" in $D[0,1]$ as follows.
(1) $\bigwedge_{j \in I}^{i} \tilde{a}_{j}=\left[\bigwedge_{j \in I} a_{j}^{-}, \bigwedge_{j \in I} a_{j}^{+}\right]$. In particular, $\tilde{a}_{1} \wedge^{i} \tilde{a}_{2}=$ $\left[a_{1}^{-} \wedge a_{2}^{-}, a_{1}^{+} \wedge a_{2}^{+}\right]$.
(2) $\bigvee_{j \in I}^{i} \tilde{a}_{j}=\left[\bigvee_{j \in I} a_{j}^{-}, \bigvee_{j \in I} a_{j}^{+}\right]$. In particular, $\widetilde{a}_{1} \vee^{i} \widetilde{a}_{2}=$ $\left[a_{1}^{-} \vee a_{2}^{-}, a_{1}^{+} \vee a_{2}^{+}\right]$.
(3) $k \widetilde{a}_{j}=\left[k a_{j}^{-}, k a_{j}^{+}\right], \forall j \in I$, where $0 \leq k \leq 1$.
(4) $\tilde{a}_{1} \leq \tilde{a}_{2} \Leftrightarrow a_{1}^{-} \leq a_{2}^{-}$and $a_{1}^{+} \leq a_{2}^{+}$.
(5) $\tilde{a}_{1}=\tilde{a}_{2} \Leftrightarrow a_{1}^{-}=a_{2}^{-}$and $a_{1}^{+}=a_{2}^{+}$.
(6) $\tilde{a}_{1}<\tilde{a}_{2} \Leftrightarrow \tilde{a}_{1} \leq \tilde{a}_{2}$ and $\tilde{a}_{1} \neq \tilde{a}_{2}$.
(7) $\tilde{a}_{1}+\tilde{a}_{2}=\left[a_{1}^{-}+a_{2}^{-}, a_{1}^{+}+a_{2}^{+}\right]$.
(8) $\tilde{a}_{1}-\tilde{a}_{2}=\left[a_{1}^{-}-a_{2}^{-}, a_{1}^{+}-a_{2}^{+}\right]$, where $\tilde{a}_{2} \leq \tilde{a}_{1}$.

Then, it is clear that $\left(D[0,1], \leq, \wedge^{i}, \vee^{i}\right)$ forms a complete lattice with $\widetilde{0}=[0,0]$ as its least element and $\widetilde{1}=[1,1]$ as its greatest element.

Let $S$ be an ordered semigroup. A mapping $\tilde{f}: S \rightarrow$ $D[0,1]$ is called an interval valued fuzzy subset of $S$, where $\tilde{f}(x)=\left[f^{-}(x), f^{+}(x)\right]$ for all $x \in S, f^{-}$and $f^{+}$are two ordinary fuzzy sets of $S$ such that $f^{-}(x) \leq f^{+}(x)$ for all $x \in S$. The set of all interval valued fuzzy sets of $S$ is denoted by $\operatorname{IVF}(S)$. Let $\tilde{f}, \tilde{g} \in I V F(S)$. Then we define
(1) $\tilde{f} \subseteq \tilde{g} \Leftrightarrow f^{-}(x) \leq g^{-}(x)$ and $f^{+}(x) \leq g^{+}(x)$ for all $x \in S$;
(2) $\tilde{f}=\tilde{g} \Leftrightarrow f^{-}(x)=g^{-}(x)$ and $f^{+}(x)=g^{+}(x)$ for all $x \in S$
(3) $(\tilde{f} \cap \tilde{g})(x)=\tilde{f}(x) \wedge^{i} \widetilde{g}(x)$ for all $x \in S$;
(4) $(\tilde{f} \cup \tilde{g})(x)=\tilde{f}(x) \vee^{i} \tilde{g}(x)$ for all $x \in S$.

One can easily show that $(\operatorname{IVF}(S), \subseteq, \cap, \cup)$ forms a complete lattice with the maximum element $\widetilde{S}$ and the minimum element $\widetilde{0}$, where the interval valued fuzzy subsets $\widetilde{S}$ and $\widetilde{0}$ of $S$ are defined by

$$
\begin{array}{lll}
\widetilde{S}: S \longrightarrow D[0,1], & x \longmapsto \widetilde{S}(x):=[1,1], & \forall x \in S, \\
\widetilde{0}: S \longrightarrow D[0,1], & x \longmapsto \widetilde{0}(x):=[0,0], \quad \forall x \in S .
\end{array}
$$


Let $(S, \cdot, \leq)$ be an ordered semigroup. For $x \in S$, we define $A_{x}:=\{(y, z) \in S \times S \mid x \leq y z\}$. For any $\tilde{f}, \widetilde{g} \in \operatorname{IVF}(S)$, the product $\tilde{f} * \widetilde{g}$ of $\tilde{f}$ and $\tilde{g}$ is defined by

$$
(\tilde{f} * \tilde{g})(x)= \begin{cases}\bigvee_{(y, z) \in A_{x}}^{i}\left[\tilde{f}(y) \wedge^{i} \tilde{g}(z)\right], & \text { if } A_{x} \neq \emptyset, \\ {[0,0],} & \text { if } A_{x} \neq \emptyset\end{cases}
$$

for all $x \in S$.

Lemma 1. Let $S$ be an ordered semigroup and $\tilde{f}, \tilde{g}, \widetilde{h} \in$ $\operatorname{IVF}(S)$. Then the following statements are true:

(1) $(\tilde{f} * \widetilde{g}) * \widetilde{h}=\widetilde{f} *(\widetilde{g} * \widetilde{h})$.

(2) If $\tilde{f} \subseteq \tilde{g}$ and $\tilde{h} \in \operatorname{IVF}(S)$, then $\tilde{f} * \tilde{h} \subseteq \widetilde{g} * \widetilde{h}, \tilde{h} * \tilde{f} \subseteq$ $\widetilde{h} * \widetilde{g}$.

Proof. The proof is similar to that of Theorem in [36].

By Lemma 1, we can easily see that this operation “*” on the set $\operatorname{IVF}(S)$ is associative and $(\operatorname{IVF}(S), *, \subseteq)$ forms an ordered semigroup.

Definition 2. An interval valued fuzzy subset $\tilde{f}$ of an ordered semigroup $S$ is called comparable if $\widetilde{f}(x)$ and $\widetilde{f}(y)$ are comparable for all $x, y \in S$; that is, $\tilde{f}(x) \geq \tilde{f}(y)$ or $\widetilde{f}(x)<$ $\tilde{f}(y)$.

Throughout this paper, let $\tilde{f}$ be a comparable interval valued fuzzy subset of $S$ unless otherwise specified.

Definition 3. Let $\tilde{f}$ be an interval valued fuzzy subset of an ordered semigroup $S$ and $\tilde{t} \in D[0,1]$. Then the crisp set

$$
\tilde{f}_{\tilde{t}}:=\{x \in S \mid \tilde{f}(x) \geq \tilde{t}\}
$$

is called a level subset of $\tilde{f}$.

Definition 4. Let $S$ be an ordered semigroup, $x \in S$ and $\tilde{t} \in D(0,1]$. An interval valued ordered fuzzy point $x_{\tilde{t}}$ of $S$ is defined by the rule that

$$
x_{\widetilde{t}}(y)= \begin{cases}\widetilde{t}, & \text { if } y \in(x], \\ {[0,0],} & \text { if } y \notin(x],\end{cases}
$$

for any $y \in S$. It is accepted that $x_{\tilde{t}}$ is a mapping from $S$ into $D[0,1]$, then an interval valued ordered fuzzy point of $S$ is an interval valued fuzzy subset of $S$. For any interval valued fuzzy subset $\tilde{f}$ of $S$, we also denote $x_{\tilde{t}} \subseteq \tilde{f}$ by $x_{\tilde{t}} \in \tilde{f}$ in the sequel.

Definition 5. An interval valued ordered fuzzy point $x_{\tilde{t}}$ of an ordered semigroup $S$ is said to be not belonging to (resp., not $k$ quasi-coincident with) an interval valued fuzzy subset $\tilde{f}$ of $S$, written as $x_{\tilde{t}} \bar{\epsilon} \tilde{f}$ (resp., $x_{\tilde{t}} \overline{q_{\tilde{k}}} \tilde{f}$ ), if $\tilde{f}(x)<\tilde{t}$ (resp., $\tilde{f}(x)+\widetilde{t}+\widetilde{k} \leq$ $[1,1])$, where $\widetilde{k} \in E D[0,1)$. If $x_{\tilde{t}} \bar{\epsilon} \widetilde{f}$ or $x_{\tilde{t}} \overline{q_{\tilde{k}}} \tilde{f}$, then we write $x_{\tilde{t}} \bar{\epsilon} \vee \overline{q_{\tilde{k}}} \tilde{f}$. The symbol $\overline{\bar{\epsilon} \vee \overline{q_{\tilde{k}}}}$ means that $\bar{\epsilon} \vee \overline{q_{\tilde{k}}}$ does not hold.
Let $A$ be a nonempty subset of an ordered semigroup $S$. We denote by $\tilde{f}_{A}$ the interval valued characteristic function of $A$, which is the mapping of $S$ into $D[0,1]$ defined by

$$
\tilde{f}_{A}(x)= \begin{cases}{[1,1],} & \text { if } x \in A, \\ {[0,0],} & \text { if } x \notin A .\end{cases}
$$

Clearly, $\widetilde{f}_{A}$ is an interval valued fuzzy subset of $S$.

The reader is referred to $[28,37,38]$ for notation and terminology not defined in this paper.

\section{Interval Valued $\left(\bar{\epsilon}, \bar{\epsilon} \vee \overline{q_{\bar{k}}}\right)$-Fuzzy Quasi-Ideals of Ordered Semigroups}

Throughout this paper, let $S$ be an ordered semigroup and let $\widetilde{k}=\left[k^{-}, k^{+}\right]$denote an arbitrary element of $E D[0,1)$ unless otherwise specified. In this section, we will introduce and study interval valued $\left(\bar{\epsilon}, \bar{\epsilon} \vee \overline{q_{\bar{k}}}\right)$-fuzzy quasi-ideals of ordered semigroups, which is a new generalized form of interval valued fuzzy quasi-ideals.

In what follows, we emphasize that any element $\tilde{t}$ in $D[0,1]$ must satisfy the following condition:

(C) $\tilde{t} \leq\left[\left(1-k^{-}\right) / 2,\left(1-k^{+}\right) / 2\right]$ or $\tilde{t}>\left[\left(1-k^{-}\right) / 2,\left(1-k^{+}\right) / 2\right]$,

where $\widetilde{k}=\left[k^{-}, k^{+}\right] \in E D[0,1)$. For any $\widetilde{f} \in \operatorname{IVF}(S)$, we say that $\tilde{f}$ satisfies the condition $(C)$ if $\widetilde{f}(x)(\in D[0,1])$ satisfies the condition $(C)$ for any $x \in S$.

In the following, we first extend the concept of fuzzy quasi-ideals to the concept of interval valued fuzzy quasiideals in an ordered semigroup as follows.

Definition 6. Let $S$ be an ordered semigroup and $\tilde{f}$ an interval valued fuzzy subset of $S$. Then $f$ is called an interval valued fuzzy quasi-ideal of $S$ if the following conditions are satisfied:

$$
\begin{aligned}
& \text { (1) } x \leq y \Rightarrow \widetilde{f}(x) \geq \widetilde{f}(y) \text { for all } x, y \in S \text {. } \\
& \text { (2) }(\widetilde{f} * \widetilde{S}) \cap(\widetilde{S} * \widetilde{f}) \subseteq \widetilde{f} \text {. }
\end{aligned}
$$

Theorem 7. Let $S$ be an ordered semigroup and $\tilde{f}$ an interval valued fuzzy subset of $S$. Then $\tilde{f}$ is an interval valued fuzzy quasi-ideal of $S$ if and only if all nonempty level subsets $\widetilde{f}_{\tilde{t}}$ of $\widetilde{f}$ are quasi-ideals of $S$ for all $\tilde{t} \in D(0,1]$.

Proof. Suppose that $\tilde{f}$ is an interval valued fuzzy quasi-ideal of $S$ and $\widetilde{t} \in D(0,1]$ such that $\widetilde{f}_{\tilde{t}} \neq \emptyset$. Let $x \in S$ be such that $x \in\left(\tilde{f}_{\tilde{t}} S\right] \cap\left(S \tilde{f}_{\tilde{t}}\right]$. Then $x \in\left(\tilde{f}_{\tilde{t}} S\right]$ and $x \in\left(S \tilde{f}_{\tilde{t}}\right]$, and we have $x \leq y z$ and $x \leq y_{1} z_{1}$ for some $y, z_{1} \in \tilde{f}_{\tilde{t}}$ and $z, y_{1} \in S$. Then $(y, z) \in A_{x}$ and $\left(y_{1}, z_{1}\right) \in A_{x}$. By hypothesis, we have

$$
\begin{aligned}
\widetilde{f}(x) & \geq((\tilde{f} * \widetilde{S}) \cap(\widetilde{S} * \widetilde{f}))(x) \\
& =(\widetilde{f} * \widetilde{S})(x) \wedge^{i}(\widetilde{S} * \widetilde{f})(x)
\end{aligned}
$$




$$
\begin{aligned}
= & \left(\bigvee_{(p, q) \in A_{x}}^{i}\left[\tilde{f}(p) \wedge^{i} \widetilde{S}(q)\right]\right) \\
& \wedge^{i}\left(\bigvee_{(u, v) \in A_{x}}^{i}\left[\widetilde{S}(u) \wedge^{i} \tilde{f}(v)\right]\right) \\
\geq & \left(\tilde{f}(y) \wedge^{i} \widetilde{S}(z)\right) \wedge^{i}\left(\tilde{S}\left(y_{1}\right) \wedge^{i} \tilde{f}\left(z_{1}\right)\right) \\
= & \left(\tilde{f}(y) \wedge^{i}[1,1]\right) \wedge^{i}\left([1,1] \wedge^{i} \tilde{f}\left(z_{1}\right)\right) \\
= & \tilde{f}(y) \wedge^{i} \tilde{f}\left(z_{1}\right) .
\end{aligned}
$$

Since $y, z_{1} \in \tilde{f}_{\tilde{t}}$, we have $\tilde{f}(y) \geq \tilde{t}$ and $\tilde{f}\left(z_{1}\right) \geq \tilde{t}$. Then $\tilde{f}(x) \geq$ $\tilde{f}(y) \wedge^{i} \widetilde{f}\left(z_{1}\right) \geq \widetilde{t} \wedge i \widetilde{t}=\widetilde{t}$, and so $x \in \tilde{f}_{\tilde{t}}$. Thus $\left(\tilde{f}_{\tilde{t}} S\right] \cap\left(S \widetilde{f}_{\tilde{t}}\right] \subseteq \widetilde{f}_{\tilde{t}}$. Furthermore, let $x \in \widetilde{f}_{\tilde{t}}, S \ni y \leq x$. Then $y \in \widetilde{f}_{\tilde{t}}$. Indeed, since $x \in \tilde{f}_{\tilde{t}}, \widetilde{f}(x) \geq \tilde{t}$, and $\tilde{f}$ is an interval valued fuzzy quasiideal of $S$, we have $\tilde{f}(y) \geq \tilde{f}(x) \geq \tilde{t}$, so $y \in \tilde{f}_{\tilde{t}}$. Therefore, $\tilde{f}_{\tilde{t}}$ is a quasi-ideal of $S$.

Conversely, assume that for every $\tilde{t} \in D(0,1]$ such that $\widetilde{f}_{\tilde{t}} \neq \emptyset$ the set $\widetilde{f}_{\tilde{t}}$ is a quasi-ideal of $S$. Let $x \in S$. Then $((\tilde{f} * \widetilde{S}) \cap$ $(\widetilde{S} * \widetilde{f}))(x) \leq \tilde{f}(x)$. In fact, if $\tilde{f}(x)<((\tilde{f} * \widetilde{S}) \cap(\widetilde{S} * \widetilde{f}))(x)$, then there exists $\tilde{t} \in D(0,1]$ such that $\widetilde{f}(x)<\widetilde{t} \leq((\widetilde{f} * \widetilde{S}) \cap$ $(\widetilde{S} * \widetilde{f}))(x)=(\tilde{f} * \widetilde{S})(x) \wedge^{i}(\widetilde{S} * \widetilde{f})(x)$, and so $(\widetilde{f} * \widetilde{S})(x) \geq$ $\tilde{t}$ and $(\widetilde{S} * \widetilde{f})(x) \geq \widetilde{t}$. Then $\bigvee_{(p, q) \in A_{x}}^{i}\left[\widetilde{f}(p) \wedge^{i} \widetilde{S}(q)\right] \geq \widetilde{t}$ and $\bigvee_{(u, v) \in A_{x}}^{i}\left[\widetilde{S}(u) \wedge^{i} \widetilde{f}(v)\right] \geq \widetilde{t}$. Since $\widetilde{f}$ is comparable, and there exist $a, b, c, d \in S$ with $(a, b) \in A_{x}$ and $(c, d) \in A_{x}$ such that $\tilde{f}(a)=\tilde{f}(a) \wedge^{i} \widetilde{S}(b) \geq \tilde{t}$ and $\tilde{f}(d)=\widetilde{S}(c) \wedge^{i} \tilde{f}(d) \geq \tilde{t}$, then $a, d \in \tilde{f}_{\tilde{t}}$ and so $a b \in \tilde{f}_{\tilde{t}} S$ and $c d \in S \tilde{f}_{\tilde{t}}$. Hence $x \in\left(\tilde{f}_{\tilde{t}} S\right]$ and $x \in\left(S \widetilde{f}_{\tilde{t}}\right]$. By hypothesis, $x \in\left(\tilde{f}_{\tilde{t}} S\right] \cap\left(S \widetilde{f}_{\tilde{t}}\right] \subseteq \tilde{f}_{\tilde{t}}$, and so $x \in \widetilde{f}_{\tilde{t}}$. Then $\widetilde{f}(x) \geq \widetilde{t}$. This is a contradiction. Thus, $(\widetilde{f} * \widetilde{S}) \cap(\widetilde{S} * \widetilde{f}) \subseteq$ $\tilde{f}$. Moreover, let $x, y \in S$. If $x \leq y$, then $\tilde{f}(x) \geq \tilde{f}(y)$. Indeed, let $\widetilde{s}=\widetilde{f}(y)$. Then $y \in \widetilde{f}_{\widetilde{s}}$. Since $\widetilde{f}_{\widetilde{s}}$ is a quasi-ideal of $S$, we have $x \in \widetilde{f}_{\tilde{s}}$. Then $\tilde{f}(x) \geq \widetilde{s}=\tilde{f}(y)$. Therefore, $\tilde{f}$ is an interval valued fuzzy quasi-ideal of $S$.

Example 8. We consider a set $S:=\{a, b, c, d, e\}$ with the following multiplication "." and the order " $\leq$ ":

$$
\begin{aligned}
& \begin{array}{l|lllll}
\cdot & a & b & c & d & e \\
\hline a & a & a & a & a & a \\
b & a & b & a & d & a \\
c & a & e & c & c & e \\
d & a & b & d & d & b \\
e & a & e & a & c & a
\end{array} \\
& \leq:=\{(a, a),(a, b),(a, c),(a, d),(a, e),(b, b), \\
& (c, c),(d, d),(e, e)\} .
\end{aligned}
$$

Then $(S, \cdot, \leq)$ is an ordered semigroup and $\{a\},\{a, b\},\{a, c\}$, $\{a, d\},\{a, e\},\{a, b, d\},\{a, c, d\},\{a, b, e\},\{a, c, f\}$ and $S$ are all quasi-ideals of $S$ (see [39]). Let $\widetilde{f}$ be an interval valued fuzzy subset of $S$ such that $\tilde{f}(a)=[0.8,0.9], \tilde{f}(b)=[0.7,0.8]$, $\widetilde{f}(c)=[0.4,0.5], \widetilde{f}(d)=[0.6,0.7]$, and $\widetilde{f}(e)=[0.4,0.5]$. Then

$$
\widetilde{f}_{\tilde{t}}= \begin{cases}S, & \text { if }[0,0]<\tilde{t} \leq[0.4,0.5], \\ \{a, b, d\}, & \text { if }[0.4,0.5]<\tilde{t} \leq[0.6,0.7], \\ \{a, b\}, & \text { if }[0.6,0.7]<\tilde{t} \leq[0.7,0.8], \\ \{a\}, & \text { if }[0.7,0.8]<\tilde{t} \leq[0.8,0.9], \\ \emptyset, & \text { if }[0.8,0.9]<\tilde{t} \leq[1,1] .\end{cases}
$$

By Theorem 7, we can verify that $\tilde{f}$ is an interval valued fuzzy quasi-ideal of $S$.

Definition 9. An interval valued fuzzy subset $\tilde{f}$ of an ordered semigroup $S$ is called an interval valued $\left(\bar{\epsilon}, \bar{\epsilon} \vee \overline{q_{\tilde{k}}}\right)$-fuzzy quasiideal of $S$, if, for all $\tilde{t}, \tilde{r} \in D(0,1]$ and $x, y, z, u, v \in S$, the following conditions hold:

$$
\begin{aligned}
& \text { (1) } x \leq y \text { and } x_{\tilde{t}} \bar{\epsilon} \tilde{f} \Rightarrow y_{\tilde{t}} \bar{\epsilon} \vee \overline{q_{\tilde{k}}} \tilde{f} . \\
& \text { (2) } x \leq y u, x \leq v z \text {, and } x_{\tilde{t} \wedge i \tilde{r}} \bar{\epsilon} \widetilde{f} \Rightarrow y_{\tilde{t}} \bar{\epsilon} \vee \overline{q_{\tilde{k}}} \tilde{f} \text { or } z_{\tilde{r}} \bar{\epsilon} \vee \overline{q_{\tilde{k}}} \tilde{f}
\end{aligned}
$$

Theorem 10. Let $S$ be an ordered semigroup and $\tilde{f}$ an interval valued fuzzy subset of $S$. Then $\tilde{f}$ is an interval valued $(\bar{\epsilon}, \bar{\epsilon} \vee$ $\left.\overline{q_{\tilde{k}}}\right)$-fuzzy quasi-ideal of $S$ if and only if $\tilde{f}$ satisfies the following conditions:

(1) $x \leq y \Rightarrow \tilde{f}(x) \vee^{i}\left[\left(1-k^{-}\right) / 2,\left(1-k^{+}\right) / 2\right] \geq \tilde{f}(y)$ for all $x, y \in S$.

(2) $\tilde{f}(x) \vee^{i}\left[\left(1-k^{-}\right) / 2,\left(1-k^{+}\right) / 2\right] \geq(\tilde{f} * \widetilde{S})(x) \wedge^{i}(\widetilde{S} * \widetilde{f})(x)$ for all $x \in S$.

Proof. Suppose that $\tilde{f}$ is an interval valued $\left(\bar{\epsilon}, \bar{\epsilon} \vee \overline{q_{\tilde{k}}}\right)$-fuzzy quasi-ideal of $S$. Let $x, y \in S$ such that $x \leq y$. If $\widetilde{f}(x) \vee^{i}[(1-$ $\left.\left.k^{-}\right) / 2,\left(1-k^{+}\right) / 2\right]<\widetilde{f}(y)=\tilde{t}$, then $\tilde{t} \in D((1-k) / 2,1]$, $x_{\tilde{t}} \bar{\epsilon} \widetilde{f}$, but $y_{\bar{t}} \overline{q_{\bar{k}}} \widetilde{f}$ does not hold. Thus, by Definition 9 , we have $y_{t} \bar{\epsilon} \tilde{f}$. It follows that $\tilde{f}(y)<\tilde{t}$, which is a contradiction with $\widetilde{f}(y)=\widetilde{t}$. Hence $\tilde{f}(x) \vee^{i}\left[\left(1-k^{-}\right) / 2,\left(1-k^{+}\right) / 2\right] \geq \tilde{f}(y)$ for all $x, y \in S$ with $x \leq y$. Furthermore, $\tilde{f}(x) \vee^{i}\left[\left(1-k^{-}\right) / 2,(1-\right.$ $\left.\left.k^{+}\right) / 2\right] \geq(\widetilde{f} * \widetilde{S})(x) \wedge^{i}(\widetilde{S} * \widetilde{f})(x)$ for all $x \in S$. Indeed, if $\widetilde{f}(x) \vee^{i}\left[\left(1-k^{-}\right) / 2,\left(1-k^{+}\right) / 2\right]<(\tilde{f} * \widetilde{S})(x) \wedge^{i}(\widetilde{S} * \widetilde{f})(x)$ for some $x \in S$, then there exists $\tilde{t} \in D((1-k) / 2,1]$ such that $\widetilde{f}(x) \vee^{i}\left[\left(1-k^{-}\right) / 2,\left(1-k^{+}\right) / 2\right]<\widetilde{t} \leq(\widetilde{f} * \widetilde{S})(x) \wedge^{i}(\widetilde{S} * \widetilde{f})(x)$. Since $(\tilde{f} * \widetilde{S})(x)>\tilde{t}>[0,0],(\widetilde{S} * \widetilde{f})(x)>\tilde{t}>[0,0]$, there exist $y, z, u, v \in S$ such that $x \leq y u$ and $x \leq v z$, and $\tilde{t} \leq \tilde{f}(y) \wedge^{i} \widetilde{S}(u)=\tilde{f}(y), \tilde{t} \leq \widetilde{S}(v) \wedge^{i} \tilde{f}(z)=\tilde{f}(z)$. Then $x_{\tilde{t}} \bar{\epsilon} \tilde{f}$, but $y_{\tilde{t}} \overline{q_{\tilde{k}}} \tilde{f}$ and $z_{\tilde{t}} \overline{q_{\tilde{k}}} \tilde{f}$ do not hold. Thus, by Definition 9, we have $y_{\bar{t}} \bar{\epsilon} \widetilde{f}$ or $z_{\tilde{t}} \bar{\epsilon} \widetilde{f}$. Then $\tilde{f}(y)<\tilde{t}$ or $\tilde{f}(z)<\widetilde{t}$, which is impossible. Therefore, $\tilde{f}(x) \vee^{i}\left[\left(1-k^{-}\right) / 2,\left(1-k^{+}\right) / 2\right] \geq$ $(\tilde{f} * \widetilde{S})(x) \wedge^{i}(\widetilde{S} * \widetilde{f})(x)$ for all $x \in S$.

Conversely, assume that the conditions (1) and (2) hold. Let $x, y \in S$ and $\tilde{t} \in D(0,1]$ be such that $x \leq y$. If $x_{\tilde{t}} \bar{\epsilon} \tilde{f}$, then 
$\tilde{f}(x)<\tilde{t}$. Since $\tilde{f}$ satisfies the condition $(C)$, we can consider the following two cases.

Case 1. If $\tilde{f}(x)>\left[\left(1-k^{-}\right) / 2,\left(1-k^{+}\right) / 2\right]$, then, by the condition (1), $\tilde{f}(y) \leq \widetilde{f}(x)<\widetilde{t}$, which means that $y_{\tilde{t}} \bar{\epsilon} \widetilde{f}$. Thus $y_{\tilde{t}} \bar{\epsilon} \vee \overline{q_{\tilde{k}}} \tilde{f}$.

Case 2. If $\widetilde{f}(x) \leq\left[\left(1-k^{-}\right) / 2,\left(1-k^{+}\right) / 2\right]$, then, by hypothesis, we have $\tilde{f}(y) \leq\left[\left(1-k^{-}\right) / 2,\left(1-k^{+}\right) / 2\right]$. If $y_{\tilde{t}} \bar{\epsilon} \tilde{f}$, then $y_{\tilde{t}} \bar{\in} \vee \overline{q_{\tilde{k}}} \tilde{f}$. Now let $y_{\tilde{t}} \in \tilde{f}$. Then, $\tilde{t} \leq \tilde{f}(y) \leq\left[\left(1-k^{-}\right) / 2,\left(1-k^{+}\right) / 2\right]$, which implies that $y_{\tilde{t}} \overline{q_{\tilde{k}}} \tilde{f}$, and thus $y_{\tilde{t}} \bar{\epsilon} \vee \overline{q_{\tilde{k}}} \tilde{f}$.

Thus, in both cases, we have $y_{\hat{t}} \bar{\epsilon} \vee \overline{q_{\tilde{k}}} \widetilde{f}$. Furthermore, let $x, y, z, u, v \in S$ and $\tilde{t}, \tilde{r} \in D(0,1]$ be such that $x \leq y u, x \leq v z$, and $x_{\tilde{t} \wedge i \widetilde{r}} \bar{\epsilon} \widetilde{f}$. Then $\widetilde{f}(x)<\widetilde{t} \wedge^{i} \widetilde{r}$, and

$$
\begin{aligned}
\tilde{f}(x) & \vee^{i}\left[\frac{1-k^{-}}{2}, \frac{1-k^{+}}{2}\right] \\
\geq & (\tilde{f} * \widetilde{S})(x) \wedge^{i}(\widetilde{S} * \tilde{f})(x) \\
= & \left(\bigvee_{(s, t) \in A_{x}}^{i}\left[\tilde{f}(s) \wedge^{i} \widetilde{S}(t)\right]\right) \\
& \wedge^{i}\left(\bigvee_{(p, q) \in A_{x}}^{i}\left[\widetilde{S}(p) \wedge^{i} \tilde{f}(q)\right]\right) \\
\geq & \left(\tilde{f}(y) \wedge^{i} \widetilde{S}(u)\right) \wedge^{i}\left(\widetilde{S}(v) \wedge^{i} \tilde{f}(z)\right) \\
= & \tilde{f}(y) \wedge^{i} \tilde{f}(z) .
\end{aligned}
$$

Since $\tilde{f}$ satisfies the condition $(C)$, we can consider the following two cases.

Case 1. If $\tilde{f}(x)>\left[\left(1-k^{-}\right) / 2,\left(1-k^{+}\right) / 2\right]$, then $\tilde{f}(y) \wedge^{i} \tilde{f}(z) \leq$ $\tilde{f}(x)<\tilde{t} \wedge^{i} \widetilde{r}$. Also, since $\tilde{f}$ is comparable, we have $\tilde{f}(y)<\tilde{t}$ or $\tilde{f}(z)<\widetilde{r}$. It thus follows that $y_{\tilde{t}} \bar{\epsilon} \widetilde{f}$ or $z_{\tilde{r}} \bar{\epsilon} \tilde{f}$, which implies that $y_{\tilde{t}} \bar{\epsilon} \vee \overline{q_{\tilde{k}}} \tilde{f}$ or $z_{\widetilde{r}} \bar{\epsilon} \vee \overline{q_{\tilde{k}}} \tilde{f}$.

Case 2. If $\tilde{f}(x y) \leq\left[\left(1-k^{-}\right) / 2,\left(1-k^{+}\right) / 2\right]$, then, by hypothesis, we have $\tilde{f}(x) \wedge^{i} \tilde{f}(y) \leq\left[\left(1-k^{-}\right) / 2,\left(1-k^{+}\right) / 2\right]$. If $y_{\tilde{t}} \bar{\epsilon} \widetilde{f}$ or $z_{\widetilde{r}} \bar{\epsilon} \tilde{f}$, then $y_{\bar{t}} \bar{\epsilon} \vee \overline{q_{\tilde{k}}} \tilde{f}$ or $z_{\tilde{r}} \bar{\epsilon} \vee \overline{q_{\tilde{k}}} \tilde{f}$. Now let $y_{\tilde{t}} \in \tilde{f}$ and $z_{\tilde{r}} \in \tilde{f}$. Then, since $\widetilde{f}$ is comparable, we have $\widetilde{t} \leq \widetilde{f}(y) \leq\left[\left(1-k^{-}\right) / 2\right.$, (1$\left.\left.k^{+}\right) / 2\right]$ or $\tilde{r} \leq \widetilde{f}(z) \leq\left[\left(1-k^{-}\right) / 2,\left(1-k^{+}\right) / 2\right]$. It follows that $y_{\bar{t}} \overline{q_{\tilde{k}}} \tilde{f}$ or $z_{\widetilde{r}} \overline{q_{\tilde{k}}} \widetilde{f}$, and thus $y_{\tilde{t}} \bar{\epsilon} \vee \overline{q_{\tilde{k}}} \tilde{f}$ or $z_{\widetilde{r}} \bar{\epsilon} \vee \overline{q_{\tilde{k}}} \tilde{f}$.

Thus, in both cases, we have $y_{\bar{t}} \bar{\epsilon} \vee \overline{q_{\tilde{k}}} \tilde{f}$ or $z_{\tilde{r}} \bar{\epsilon} \vee \overline{q_{\tilde{k}}} \tilde{f}$. Therefore, $\tilde{f}$ is an interval valued $\left(\bar{\epsilon}, \bar{\epsilon} \vee \overline{q_{\tilde{k}}}\right)$-fuzzy quasi-ideal of $S$.

From Theorem 10, we can see that the interval valued $\left(\bar{\epsilon}, \bar{\epsilon} \vee \overline{q_{\tilde{k}}}\right)$-fuzzy quasi-ideal is characterized in terms of the multiplications $\tilde{f} * \widetilde{S}$ and $\widetilde{S} * \widetilde{f}$. A natural question is if an interval valued $\left(\bar{\epsilon}, \bar{\epsilon} \vee \overline{q_{\tilde{k}}}\right)$-fuzzy quasi-ideal $\widetilde{f}$ can be defined using only the interval valued fuzzy subset $\tilde{f}$ itself. The theorems below give the answer.
Theorem 11. Let $S$ be an ordered semigroup. Then an interval valued fuzzy subset $\tilde{f}$ of $S$ is an interval valued $\left(\bar{\epsilon}, \bar{\epsilon} \vee \overline{q_{\tilde{k}}}\right)$ fuzzy quasi-ideal of $S$ if and only if the following conditions are satisfied:

(1) $x \leq y \Rightarrow \tilde{f}(x) \vee^{i}\left[\left(1-k^{-}\right) / 2,\left(1-k^{+}\right) / 2\right] \geq \tilde{f}(y)$ for all $x, y \in S$.

(2) $x \leq y u$ and $x \leq v z \Rightarrow \widetilde{f}(x) \vee^{i}\left[\left(1-k^{-}\right) / 2,\left(1-k^{+}\right) / 2\right] \geq$ $\widetilde{f}(y) \wedge^{i} \widetilde{f}(z)$ for all $x, y, z, u, v \in S$.

Proof. $\Rightarrow$ : Let $x, y, z, u, v \in S$ be such that $x \leq y u$ and $x \leq v z$. Since $\tilde{f}$ is an interval valued $\left(\bar{\epsilon}, \bar{\epsilon} \vee \overline{q_{\tilde{k}}}\right)$-fuzzy quasi-ideal of $S$ and $x \in S$, by Theorem 10 , we have

$$
\widetilde{f}(x) \vee^{i}\left[\frac{1-k^{-}}{2}, \frac{1-k^{+}}{2}\right] \geq(\tilde{f} * \widetilde{S})(x) \wedge^{i}(\widetilde{S} * \widetilde{f})(x) .
$$

Since $x \leq y u$, we have $(y, u) \in A_{x}$; then

$$
\begin{aligned}
(\tilde{f} * \widetilde{S})(x) & =\bigvee_{(s, t) \in A_{x}}^{i}\left[\tilde{f}(s) \wedge^{i} \widetilde{S}(t)\right] \\
& \geq \tilde{f}(y) \wedge^{i} \widetilde{S}(u)=\tilde{f}(y) \wedge^{i}[1,1]=\tilde{f}(y) .
\end{aligned}
$$

By $x \leq v z$ in a similar way we can get $(\widetilde{S} * \widetilde{f})(x) \geq \widetilde{f}(z)$. It thus follows that $\tilde{f}(x) \vee^{i}\left[\left(1-k^{-}\right) / 2,\left(1-k^{+}\right) / 2\right] \geq \tilde{f}(y) \wedge^{i} \tilde{f}(z)$. $\Leftarrow$ : Assume that the conditions (1) and (2) hold. Let $x \in S$. Then $\widetilde{f}(x) \vee^{i}\left[\left(1-k^{-}\right) / 2,\left(1-k^{+}\right) / 2\right] \geq(\widetilde{f} * \widetilde{S})(x) \wedge^{i}(\widetilde{S} * \widetilde{f})(x)$. Indeed, if $A_{x}=\emptyset$, then $(\widetilde{f} * \widetilde{S})(x) \wedge^{i}(\widetilde{S} * \widetilde{f})(x)=[0,0]$, and

$$
\begin{aligned}
& (\tilde{f}) \widetilde{S})(x) \wedge^{i}(\widetilde{S} * \tilde{f})(x) \\
& \quad=[0,0] \\
& \quad \leq \tilde{f}(x) \vee^{i}\left[\frac{1-k^{-}}{2}, \frac{1-k^{+}}{2}\right] .
\end{aligned}
$$

Let $A_{x} \neq \emptyset$. Then

$$
\begin{aligned}
& (\tilde{f} * \widetilde{S})(x)=\bigvee_{(s, t) \in A_{x}}^{i}\left[\tilde{f}(s) \wedge^{i} \widetilde{S}(t)\right], \\
& (\widetilde{S} * \widetilde{f})(x)=\bigvee_{(p, q) \in A_{x}}^{i}\left[\widetilde{S}(p) \wedge^{i} \tilde{f}(q)\right] .
\end{aligned}
$$

Since $\tilde{f}$ is comparable and satisfies the condition $(C)$, we can consider the following two cases.

Case 1. If $\tilde{f}(x) \vee^{i}\left[\left(1-k^{-}\right) / 2,\left(1-k^{+}\right) / 2\right] \geq(\tilde{f} * \widetilde{S})(x)$, then $\widetilde{f}(x) \vee^{i}\left[\left(1-k^{-}\right) / 2,\left(1-k^{+}\right) / 2\right] \geq(\widetilde{f} * \widetilde{S})(x) \wedge^{i}(\widetilde{S} * \widetilde{f})(x)$.

Case 2. Let $\tilde{f}(x) \vee^{i}\left[\left(1-k^{-}\right) / 2,\left(1-k^{+}\right) / 2\right]<(\tilde{f} * \widetilde{S})(x)$. Then, by (14), there exists $(y, u) \in A_{x}$ such that $\tilde{f}(x) \vee^{i}\left[\left(1-k^{-}\right) / 2,(1-\right.$ $\left.\left.k^{+}\right) / 2\right]<\tilde{f}(y) \wedge^{i} \widetilde{S}(u)$. Since $\tilde{f}(y) \wedge^{i} \widetilde{S}(u)=\widetilde{f}(y)$, we have

$$
\tilde{f}(x) \vee^{i}\left[\frac{1-k^{-}}{2}, \frac{1-k^{+}}{2}\right]<\tilde{f}(y) .
$$


So we can show that $\tilde{f}(x) \vee^{i}\left[\left(1-k^{-}\right) / 2,\left(1-k^{+}\right) / 2\right] \geq$ $\widetilde{S}(v) \wedge^{i} \tilde{f}(z)$ for any $(v, z) \in A_{x}$. In fact, since $(y, u) \in A_{x}$, we have $y, u \in S$ and $x \leq y u$. Since $(v, z) \in A_{x}$, we have $v, z \in S$ and $x \leq v z$. Since there exist $x, y, z, u, v \in S$ such that $x \leq y u$ and $x \leq v z$, by hypothesis, we have

$$
\tilde{f}(x) \vee^{i}\left[\frac{1-k^{-}}{2}, \frac{1-k^{+}}{2}\right] \geq \tilde{f}(y) \wedge^{i} \tilde{f}(z) .
$$

Since $\tilde{f}$ is comparable, we have $\widetilde{f}(y) \wedge^{i} \widetilde{f}(z)=\widetilde{f}(y)$ or $\widetilde{f}(y) \wedge^{i} \widetilde{f}(z)=\tilde{f}(z)$. If $\widetilde{f}(y) \wedge^{i} \widetilde{f}(z)=\widetilde{f}(y)$, then $\widetilde{f}(x) \vee^{i}[(1-$ $\left.\left.k^{-}\right) / 2,\left(1-k^{+}\right) / 2\right] \geq \widetilde{f}(y)$, which is impossible by (15). Hence we have

$$
\tilde{f}(x) \vee^{i}\left[\frac{1-k^{-}}{2}, \frac{1-k^{+}}{2}\right] \geq \widetilde{f}(z)=\widetilde{S}(v) \wedge^{i} \tilde{f}(z),
$$

for any $(v, z) \in A_{x}$. Then we have

$$
\begin{aligned}
\tilde{f}(x) & \wedge^{i}\left[\frac{1-k^{-}}{2}, \frac{1-k^{+}}{2}\right] \\
& \geq \bigvee_{(v, z) \in A_{x}}^{i}\left[\widetilde{S}(v) \wedge^{i} \widetilde{f}(z)\right]=(\widetilde{S} * \widetilde{f})(x) \\
& \geq(\widetilde{f} * \widetilde{S})(x) \wedge^{i}(\widetilde{S} * \widetilde{f})(x),
\end{aligned}
$$

and the proof is completed by Theorem 10 .

Theorem 12. Let $S$ be an ordered semigroup. Then an interval valued fuzzy subset $\tilde{f}$ of $S$ is an interval valued $\left(\bar{\epsilon}, \bar{\epsilon} \vee \overline{q_{\tilde{k}}}\right)$ fuzzy quasi-ideal of $S$ if and only if the following conditions are satisfied:

(1) $x \leq y \Rightarrow \tilde{f}(x) \vee^{i}\left[\left(1-k^{-}\right) / 2,\left(1-k^{+}\right) / 2\right] \geq \tilde{f}(y)$.

(2) $x \leq y u$ and $x \leq v z \Rightarrow \tilde{f}(x) \vee^{i}\left[\left(1-k^{-}\right) / 2,\left(1-k^{+}\right) / 2\right] \geq$ $\left(\tilde{f}(y) \wedge^{i} \tilde{f}(z)\right) \vee^{i}\left(\tilde{f}(v) \wedge^{i} \tilde{f}(u)\right)$ for all $x, y, z, u, v \in S$.

Proof. The proof is straightforward verification by Theorem 11 and we omit it.

By Theorem 10, we can easily observe that every interval valued fuzzy quasi-ideal of an ordered semigroup $S$ is an interval valued $\left(\bar{\epsilon}, \bar{\epsilon} \vee \overline{q_{\bar{k}}}\right)$-fuzzy quasi-ideal of $S$. However, the converse is not true, in general, as shown in the following example.

Example 13. Consider the ordered semigroup $S$ given in Example 8 and define an interval valued fuzzy subset $\tilde{f}$ of $S$ by $\widetilde{f}(a)=[0.4,0.5], \widetilde{f}(b)=[0.3,0.4], \widetilde{f}(c)=[0.2,0.3]$, and $\widetilde{f}(d)=\widetilde{f}(e)=[0.1,0.2]$. Then

$$
\widetilde{f}_{\tilde{t}}= \begin{cases}S, & \text { if }[0,0]<\tilde{t} \leq[0.1,0.2], \\ \{a, b, c\}, & \text { if }[0.1,0.2]<\widetilde{t} \leq[0.2,0.3], \\ \{a, b\}, & \text { if }[0.2,0.3]<\tilde{t} \leq[0.3,0.4], \\ \{a\}, & \text { if }[0.3,0.4]<\widetilde{t} \leq[0.4,0.5], \\ \emptyset, & \text { if }[0.4,0.5]<\tilde{t} \leq[1,1] .\end{cases}
$$

By Theorem 11, we can easily show that $\tilde{f}$ is an interval valued $\left(\bar{\epsilon}, \bar{\epsilon} \vee \overline{q_{\tilde{k}}}\right)$-fuzzy quasi-ideal of $S$ for any $\widetilde{k} \in E D[0,0.4]$. But $\tilde{f}$ is not an interval valued fuzzy quasi-ideal of $S$. In fact, since $\{a, b, c\}$ is not a quasi-ideal of $S$, we deduce that $\tilde{f}$ is not an interval valued fuzzy quasi-ideal of $S$ by Theorem 7 .

In the following theorem, we give a condition for an interval valued $\left(\bar{\epsilon}, \bar{\epsilon} \vee \overline{q_{\tilde{k}}}\right)$-fuzzy quasi-ideal of an ordered semigroup $S$ to be an interval valued fuzzy quasi-ideal of $S$.

Theorem 14. Let $S$ be an ordered semigroup and $\tilde{f}$ an interval valued $\left(\bar{\epsilon}, \bar{\epsilon} \vee \overline{q_{\tilde{k}}}\right)$-fuzzy quasi-ideal of $S$. If $\tilde{f}(x) \geq[(1-$ $\left.\left.k^{-}\right) / 2,\left(1-k^{+}\right) / 2\right]$ for all $x \in S$, then $\widetilde{f}$ is an interval valued fuzzy quasi-ideal of $S$.

Proof. It is obvious by Theorem 10 .

Theorem 15. Let $S$ be an ordered semigroup. If $\tilde{k}, \widetilde{r} \in \operatorname{ED}[0,1)$ and $\widetilde{k}>\widetilde{r}$, then every interval valued $\left(\bar{\epsilon}, \bar{\epsilon} \vee \overline{q_{\tilde{k}}}\right)$-fuzzy quasiideal of $S$ is an interval valued $\left(\bar{\epsilon}, \bar{\epsilon} \vee \overline{q_{\tilde{r}}}\right)$-fuzzy quasi-ideal of $S$.

Proof. The proof is straightforward and we omit it.

The following example shows that the converse of Theorem 15 does not hold, in general.

Example 16. Consider the ordered semigroup $S$ given in Example 8 and define an interval valued fuzzy subset $\widetilde{f}$ of $S$ by $\widetilde{f}(a)=[0.4,0.5], \widetilde{f}(b)=[0.3,0.4], \widetilde{f}(c)=[0.2,0.3]$, and $\tilde{f}(d)=\tilde{f}(e)=[0.1,0.2]$. Then $\tilde{f}$ is an interval valued $\left(\bar{\epsilon}, \bar{\epsilon} \vee \overline{q_{\widetilde{r}}}\right)$-fuzzy quasi-ideal of $S$ for any $\widetilde{r} \in E D[0,0.4]$. But $\tilde{f}$ is not an interval valued $\left(\bar{\epsilon}, \bar{\epsilon} \vee \overline{q_{\tilde{k}}}\right)$-fuzzy quasi-ideal of $S$, where $\widetilde{k}=[0.6,0.6]$. In fact, since $d \leq d=b d$ and $d \leq d=d c$,

$$
\begin{aligned}
\tilde{f} & (d) \vee^{i}\left[\frac{1-k^{-}}{2}, \frac{1-k^{+}}{2}\right] \\
& =[0.1,0.2] \vee^{i}\left[\frac{1-k^{-}}{2}, \frac{1-k^{+}}{2}\right] \\
& =[0.2,0.2]<[0.2,0.3]=\tilde{f}(b) \wedge^{i} \tilde{f}(c),
\end{aligned}
$$

where $\widetilde{k}=[0.6,0.6]$. Thus, by Theorem 11, $\tilde{f}$ is not an interval valued $\left(\bar{\epsilon}, \bar{\epsilon} \vee \overline{q_{[0.6,0.6]}}\right)$-fuzzy quasi-ideal of $S$.

Now we will characterize the interval valued $\left(\bar{\epsilon}, \bar{\epsilon} \vee \overline{q_{\widetilde{k}}}\right)$ fuzzy quasi-ideals by using their level subsets.

Theorem 17. Let $S$ be an ordered semigroup and $\tilde{f}$ an interval valued fuzzy subset of S. Then $\tilde{f}$ is an interval valued $\left(\bar{\epsilon}, \bar{\epsilon} \vee \overline{q_{\tilde{k}}}\right)$ fuzzy quasi-ideal of $S$ if and only if the level subset $\tilde{f}_{\tilde{t}}$ of $\tilde{f}$ is a quasi-ideal of $S$ for all $\widetilde{t} \in D((1-k) / 2,1]$ with $\widetilde{f}_{\tilde{t}} \neq \emptyset$.

Proof. Suppose that $\tilde{f}$ is an interval valued $\left(\bar{\epsilon}, \bar{\epsilon} \vee \overline{q_{\tilde{k}}}\right)$-fuzzy quasi-ideal of $S$. Let $x, y \in S, x \leq y$ be such that $y \in \widetilde{f}_{\tilde{t}}$ 
for some $\tilde{t} \in D((1-k) / 2,1]$. Then $\tilde{f}(y) \geq \widetilde{t}$. It follows from Theorem 11(1) that

$$
\tilde{f}(x) \vee^{i}\left[\frac{1-k^{-}}{2}, \frac{1-k^{+}}{2}\right] \geq \tilde{f}(y) \geq \tilde{t} .
$$

Note that $\tilde{t} \in D((1-k) / 2,1]$, and we conclude that $\tilde{f}(x) \geq \tilde{t}$, which implies that $x \in \tilde{f}_{\tilde{t}}$. Furthermore, let $x \in\left(\tilde{f}_{\tilde{t}} S\right] \cap\left(S \tilde{f}_{\tilde{t}}\right]$ for some $t \in D((1-k) / 2,1]$. Then $x \in\left(\tilde{f}_{\tilde{t}} S\right]$ and $x \in\left(S \tilde{f}_{\tilde{t}}\right]$, and there exist $y, z \in \tilde{f}_{\tilde{t}}$ and $u, v \in S$ such that $x \leq y u$ and $x \leq v z$. Then $\widetilde{f}(y) \geq \widetilde{t}$ and $\tilde{f}(z) \geq \widetilde{t}$. It follows from Theorem 11(2) that

$$
\widetilde{f}(x) \vee^{i}\left[\frac{1-k^{-}}{2}, \frac{1-k^{+}}{2}\right] \geq \widetilde{f}(y) \wedge^{i} \widetilde{f}(z) \geq \widetilde{t} \wedge^{i} \widetilde{t}=\widetilde{t} .
$$

It implies that $x \in \tilde{f}_{\tilde{t}}$, according to $t \in D((1-k) / 2,1]$. Therefore, $\tilde{f}_{\tilde{t}}$ is a quasi-ideal of $S$ for all $\tilde{t} \in D((1-k) / 2,1]$ with $\tilde{f}_{\tilde{t}} \neq \emptyset$.

Conversely, assume that $\tilde{f}_{\tilde{t}}$ is a quasi-ideal of $S$ for all $\tilde{t} \in D((1-k) / 2,1]$ with $\widetilde{f}_{\tilde{t}} \neq \emptyset$. If there exist $x, y \in S$ with $x \leq y$ such that $\widetilde{f}(x) \vee^{i}\left[\left(1-k^{-}\right) / 2,\left(1-k^{+}\right) / 2\right]<\tilde{f}(y)=\tilde{r}$, then $\widetilde{r} \in D((1-k) / 2,1], \tilde{f}(x)<\widetilde{r}$, and $y \in \tilde{f}_{\tilde{r}}$. Since $\tilde{f}_{\tilde{r}}$ is a quasi-ideal of $S$ for any $\tilde{r} \in D((1-k) / 2,1]$ and $x \leq y$, we have $x \in \widetilde{f}_{\tilde{r}}$ and $\widetilde{f}(x) \geq \widetilde{r}$, which contradicts with $\tilde{f}(x)<\tilde{r}$. Hence $\widetilde{f}(x) \vee^{i}\left[\left(1-k^{-}\right) / 2,\left(1-k^{+}\right) / 2\right] \geq \widetilde{f}(y)$. Furthermore, let $x, y, z, u, v \in S$ such that $x \leq y u$ and $x \leq v z$. If possible, $\tilde{f}(x) \vee^{i}\left[\left(1-k^{-}\right) / 2,\left(1-k^{+}\right) / 2\right]<\widetilde{f}(y) \wedge^{i} \widetilde{f}(z)$ for some $x, y, z, u, v \in S$ such that $x \leq y u$ and $x \leq v z$, then there exists $\widetilde{s} \in D((1-k) / 2,1]$ such that $\tilde{f}(x) \vee^{i}\left[\left(1-k^{-}\right) / 2,\left(1-k^{+}\right) / 2\right]<$ $\widetilde{s} \leq \widetilde{f}(y) \wedge^{i} \widetilde{f}(z)$. Then $y, z \in \widetilde{f}_{\widetilde{s}}$. By hypothesis, we have $x \in\left(\widetilde{f}_{\tilde{s}} S\right] \cap\left(S \widetilde{f}_{\tilde{s}}\right] \subseteq \widetilde{f}_{\tilde{s}}$, and so $\widetilde{f}(x) \geq \widetilde{\mathcal{s}}$, which is impossible. Thus $\widetilde{f}(x) \vee^{i}\left[\left(1-k^{-}\right) / 2,\left(1-k^{+}\right) / 2\right] \geq \widetilde{f}(y) \wedge^{i} \widetilde{f}(z)$. Therefore, $\tilde{f}$ is an interval valued $\left(\bar{\epsilon}, \bar{\epsilon} \vee \overline{q_{\tilde{k}}}\right)$-fuzzy quasi-ideal of $S$ by Theorem 11.

Theorem 18. Let $Q$ be a nonempty subset of an ordered semigroup $S$ and $\tilde{f}$ an interval valued fuzzy subset of $S$ defined by

$$
\tilde{f}(x)= \begin{cases}{[1,1],} & \text { if } x \in Q, \\ \widetilde{r}, & \text { if } x \notin Q,\end{cases}
$$

for any $x \in S$, where $\tilde{r} \in D[0,(1-k) / 2]$. Then $Q$ is a quasiideal of $S$ if and only if $\tilde{f}$ is an interval valued $\left(\bar{\epsilon}, \bar{\epsilon} \vee \overline{q_{\tilde{k}}}\right)$-fuzzy quasi-ideal of $S$.

Proof. Suppose that $Q$ is a quasi-ideal of $S$ and let $\tilde{f}_{\tilde{t}}$ be the level subset of $\tilde{f}$. Then it is easy to see that $\tilde{f}_{\tilde{t}}=Q$ for all $\tilde{t} \in D((1-k) / 2,1]$. It thus follows from Theorem 17 that $\tilde{f}$ is an interval valued $\left(\bar{\epsilon}, \bar{\epsilon} \vee \overline{q_{\widetilde{k}}}\right)$-fuzzy quasi-ideal of $S$.

Conversely, assume that $\tilde{f}$ is an interval valued $\left(\bar{\epsilon}, \bar{\epsilon} \vee \overline{q_{\bar{k}}}\right)$ fuzzy quasi-ideal of $S$. Let $x \in S, y \in Q$ and $x \leq y$. Then $\tilde{f}(y)=[1,1]$. By Theorem 11, $\tilde{f}(x) \vee^{i}\left[\left(1-k^{-}\right) / 2,\left(1-k^{+}\right) / 2\right] \geq$ $\widetilde{f}(y)=[1,1]$. Thus, by the definition of $\widetilde{f}$, we have $\widetilde{f}(x)=$ $[1,1]$, that is, $x \in Q$. Furthermore, let $x \in(Q S] \cap(S Q]$. Then $x \in(\mathrm{QS}]$ and $x \in(S Q]$, and there exist $y, z \in$ Qand $u, v \in S$ such that $x \leq y u$ and $x \leq v z$. Then $\widetilde{f}(y)=\widetilde{f}(z)=[1,1]$. It thus follows from Theorem 11 that $\widetilde{f}(x) \mathrm{V}^{i}\left[\left(1-k^{-}\right) / 2,(1-\right.$ $\left.\left.k^{+}\right) / 2\right] \geq \tilde{f}(y) \wedge^{i} \tilde{f}(z)=[1,1]$, which implies that $\widetilde{f}(x)=$ $[1,1]$, that is, $x \in Q$. Therefore, $Q$ is a quasi-ideal of $S$.

Lemma 19. Let $S$ be an ordered semigroup and $\emptyset \neq Q \subseteq S$. Then $Q$ is a quasi-ideal of $S$ if and only if the interval valued characteristic function $\widetilde{f}_{\mathrm{Q}}$ of $Q$ is an interval valued $\left(\bar{\epsilon}, \bar{\epsilon} \vee \overline{q_{\tilde{k}}}\right)$ fuzzy quasi-ideal of S.

Proof. The proof is straightforward by Theorem 18 .

Theorem 20. Let $\left\{\tilde{f}_{j} \mid j \in I\right\}$ be a family of interval valued $\left(\bar{\epsilon}, \bar{\epsilon} \vee \overline{q_{\bar{k}}}\right)-$ fuzzy quasi-ideals of an ordered semigroup $S$. Then $\tilde{f}:=\bigcap_{j \in I} \widetilde{f}_{j}$ is an interval valued $\left(\bar{\epsilon}, \bar{\epsilon} \vee \overline{q_{\tilde{k}}}\right)$-fuzzy quasi-ideal of $S$, where $\left(\bigcap_{j \in I} \tilde{f}_{j}\right)(x)=\bigwedge_{j \in I}^{i}\left(\tilde{f}_{j}(x)\right)$.

Proof. Let $x, y, z, u, v \in S$ such that $x \leq y u$ and $x \leq v z$. Then, since each $f_{i}(i \in I)$ is an interval valued $\left(\bar{\epsilon}, \bar{\epsilon} \vee \overline{q_{\bar{k}}}\right)$-fuzzy quasi-ideal of $S$, we have

$$
\begin{aligned}
\tilde{f}(x) & \vee^{i}\left[\frac{1-k^{-}}{2}, \frac{1-k^{+}}{2}\right] \\
& =\left(\bigcap_{j \in I} \tilde{f}_{j}\right)(x) \vee^{i}\left[\frac{1-k^{-}}{2}, \frac{1-k^{+}}{2}\right] \\
& =\bigwedge_{j \in I}^{i}\left(\tilde{f}_{j}(x)\right) \vee^{i}\left[\frac{1-k^{-}}{2}, \frac{1-k^{+}}{2}\right] \\
& =\bigwedge_{j \in I}^{i}\left(\tilde{f}_{j}(x) \vee^{i}\left[\frac{1-k^{-}}{2}, \frac{1-k^{+}}{2}\right]\right) \\
& \geq \bigwedge_{j \in I}^{i}\left(\tilde{f}_{j}(y) \wedge^{i} \tilde{f}_{j}(z)\right) \\
& =\left(\bigwedge_{j \in I}^{i} \tilde{f}_{j}(y)\right) \wedge^{i}\left(\bigwedge_{j \in I}^{i} \tilde{f}_{j}(z)\right) \\
& =\left(\bigcap_{j \in I} \tilde{f}_{j}\right)(y) \wedge^{i}\left(\bigcap_{j \in I} \tilde{f}_{j}\right)(z) \\
& =\tilde{f}(y) \wedge^{i} \tilde{f}(z) .
\end{aligned}
$$

Furthermore, if $x \leq y$, then $\widetilde{f}(x) \vee^{i}\left[\left(1-k^{-}\right) / 2,\left(1-k^{+}\right) / 2\right] \geq$ $\tilde{f}(y)$. Indeed, since each $\tilde{f}_{j}(j \in I)$ is an interval valued $(\bar{\epsilon}, \bar{\epsilon} \mathrm{V}$ $\left.\overline{q_{\widetilde{k}}}\right)$-fuzzy quasi-ideal of $S$, we have $\tilde{f}_{j}(x) \vee^{i}\left[\left(1-k^{-}\right) / 2,(1-\right.$ $\left.\left.k^{+}\right) / 2\right] \geq \tilde{f}_{j}(y)$ for all $j \in I$. Thus

$$
\begin{aligned}
\tilde{f}(x) & \vee^{i}\left[\frac{1-k^{-}}{2}, \frac{1-k^{+}}{2}\right] \\
= & \left(\bigcap_{j \in I} \tilde{f}_{j}\right)(x) \vee^{i}\left[\frac{1-k^{-}}{2}, \frac{1-k^{+}}{2}\right]
\end{aligned}
$$




$$
\begin{aligned}
& =\bigwedge_{j \in I}^{i}\left(\tilde{f}_{j}(x)\right) \vee^{i}\left[\frac{1-k^{-}}{2}, \frac{1-k^{+}}{2}\right] \\
& =\bigwedge_{j \in I}^{i}\left(\tilde{f}_{j}(x) \vee^{i}\left[\frac{1-k^{-}}{2}, \frac{1-k^{+}}{2}\right]\right) \\
& \geq \bigwedge_{j \in I}^{i}\left(\tilde{f}_{j}(y)\right)=\left(\bigcap_{j \in I} \tilde{f}_{j}\right)(y)=\tilde{f}(y) .
\end{aligned}
$$

Therefore, $\tilde{f}$ is an interval valued $\left(\bar{\epsilon}, \bar{\epsilon} \vee \overline{q_{\tilde{k}}}\right)$-fuzzy quasi-ideal of $S$ by Theorem 11 .

Suppose that $\left\{\tilde{f}_{j} \mid j \in I\right\}$ is a family of interval valued $\left(\bar{\epsilon}, \bar{\epsilon} \vee \overline{q_{\tilde{k}}}\right)$-fuzzy quasi-ideals of an ordered semigroup $S$. Is it true that $\bigcup_{j \in I} \widetilde{f}_{j}$ is an interval valued $\left(\bar{\epsilon}, \bar{\epsilon} \vee \overline{q_{\widetilde{k}}}\right)$-fuzzy quasiideal of $S$, where $\left(\bigcup_{j \in I} \widetilde{f}_{j}\right)(x)=\bigvee_{j \in I}^{i}\left(\widetilde{f}_{j}(x)\right)$ ?. The following example gives a negative answer to the above question.

Example 21. We consider the ordered semigroup $S:=$ $\{a, b, c, d\}$ defined by the following multiplication "." and the order " $\leq$ ":

$$
\begin{aligned}
& \begin{array}{l|llll}
\cdot & a & b & c & d \\
\hline a & a & a & a & a \\
b & a & a & d & a \\
c & a & a & a & a \\
d & a & a & a & a
\end{array} \\
& \leq:=\{(a, a),(a, d),(b, b),(c, c),(d, d)\} .
\end{aligned}
$$

Let $\widetilde{f}_{1}$ and $\widetilde{f}_{2}$ be two interval valued fuzzy subsets of $S$ such that

$$
\begin{array}{ll}
\tilde{f}_{1}(a)=\tilde{f}_{1}(b)=[0.6,0.7], & \tilde{f}_{1}(c)=\tilde{f}_{1}(d)=[0,0] ; \\
\tilde{f}_{2}(a)=\tilde{f}_{2}(c)=[0.6,0.7], & \tilde{f}_{2}(b)=\tilde{f}_{2}(d)=[0,0] .
\end{array}
$$

Then, by Theorem 11, $\tilde{f}_{1}$ and $\tilde{f}_{2}$ are both interval valued $(\bar{\epsilon}, \bar{\epsilon} \mathrm{V}$ $\left.\overline{q_{\tilde{k}}}\right)$-fuzzy quasi-ideals of $S$ for any $\widetilde{k} \in \operatorname{ED}[0,1)$. But $\widetilde{f}_{1} \cup \widetilde{f}_{2}$ is not an interval valued $\left(\bar{\epsilon}, \bar{\epsilon} \vee \overline{q_{\tilde{k}}}\right)$-fuzzy quasi-ideal of $S$ for any $\widetilde{k} \in E D[0,1)$. In fact, since $d \leq d=b c$ and $d \leq d=b c$,

$$
\begin{aligned}
& \left(\tilde{f}_{1} \cup \tilde{f}_{2}\right)(d) \vee^{i}\left[\frac{1-k^{-}}{2}, \frac{1-k^{+}}{2}\right] \\
& =\tilde{f}_{1}(d) \vee^{i} \tilde{f}_{2}(d) \vee^{i}\left[\frac{1-k^{-}}{2}, \frac{1-k^{+}}{2}\right] \\
& =\left[\frac{1-k^{-}}{2}, \frac{1-k^{+}}{2}\right]<[0.6,0.7] \\
& =\left(\tilde{f}_{1} \cup \tilde{f}_{2}\right)(b) \wedge^{i}\left(\tilde{f}_{1} \cup \tilde{f}_{2}\right)(c) .
\end{aligned}
$$

By Theorem 11, $\tilde{f}_{1} \cup \tilde{f}_{2}$ is not an interval valued $\left(\bar{\epsilon}, \bar{\epsilon} \vee \overline{q_{\tilde{k}}}\right)$ fuzzy quasi-ideal of $S$.

The following theorem can be obtained under the assumption of an additional condition.
Theorem 22. Let $\left\{\widetilde{f}_{j} \mid j \in I\right\}$ be a family of interval valued $\left(\bar{\epsilon}, \bar{\epsilon} \vee \overline{q_{\tilde{k}}}\right)$-fuzzy quasi-ideals of an ordered semigroup $S$. Then $\widetilde{f}:=\bigcup_{j \in I} \widetilde{f}_{j}$ is an interval valued $\left(\bar{\epsilon}, \bar{\epsilon} \vee \overline{q_{\tilde{k}}}\right)$-fuzzy quasi-ideal of $S$, where $\left(\bigcup_{j \in I} \tilde{f}_{j}\right)(x)=\vee_{j \in I}^{i}\left(\tilde{f}_{j}(x)\right)$.

Proof. Let $x, y, z, u, v \in S$ such that $x \leq y u$ and $x \leq v z$. Then we have

$$
\begin{aligned}
\tilde{f}(x) & \vee^{i}\left[\frac{1-k^{-}}{2}, \frac{1-k^{+}}{2}\right] \\
& =\left(\bigcup_{j \in I} \tilde{f}_{j}\right)(x) \vee^{i}\left[\frac{1-k^{-}}{2}, \frac{1-k^{+}}{2}\right] \\
& =\bigvee_{j \in I}^{i}\left(\tilde{f}_{j}(x)\right) \vee^{i}\left[\frac{1-k^{-}}{2}, \frac{1-k^{+}}{2}\right] \\
& =\bigvee_{j \in I}^{i}\left(\tilde{f}_{j}(x) \vee^{i}\left[\frac{1-k^{-}}{2}, \frac{1-k^{+}}{2}\right]\right) \\
& \geq \bigvee_{j \in I}^{i}\left(\tilde{f}_{j}(y) \wedge^{i} \tilde{f}_{j}(z)\right)\left(\text { Since } \tilde{f}_{j}\right. \text { is an intervalued } \\
& =\tilde{f}(y) \wedge^{i} \tilde{f}(z) . \\
& =\left(\bigvee_{j \in I}^{i} \tilde{f}_{j}(y)\right) \wedge^{i}\left(\bigvee_{j \in I}^{i} \tilde{f}_{j}(z)\right)
\end{aligned}
$$

In the following we show that $(*)$ holds. It is obvious that $\bigvee_{j \in I}^{i}\left(\tilde{f}_{j}(y) \wedge^{i} \tilde{f}_{j}(z)\right) \leq\left(\bigvee_{j \in I}^{i} \tilde{f}_{j}(y)\right) \wedge^{i}\left(\bigvee_{j \in I}^{i} \tilde{f}_{j}(z)\right)$. Assume that $\bigvee_{j \in I}^{i}\left(\tilde{f}_{j}(y) \wedge^{i} \tilde{f}_{j}(z)\right) \neq\left(\bigvee_{j \in I}^{i} \widetilde{f}_{j}(y)\right) \wedge^{i}\left(\bigvee_{j \in I}^{i} \widetilde{f}_{j}(z)\right)$. Then there exists $\tilde{r} \in D(0,1)$ such that $\bigvee_{j \in I}^{i}\left(\tilde{f}_{j}(y) \wedge^{i} \widetilde{f}_{j}(z)\right)<\widetilde{r}<\left(\bigvee_{j \in I}^{i}\right.$ $\left.\widetilde{f}_{j}(y)\right) \wedge^{i}\left(\bigvee_{j \in I}^{i} \widetilde{f}_{j}(z)\right)$. Since $\widetilde{f}_{m} \subseteq \widetilde{f}_{n}$ or $\widetilde{f}_{n} \subseteq \widetilde{f}_{m}$ for all $m, n \in$ $I$, there exists $l \in I$ such that $\widetilde{r}<\tilde{f}_{l}(y) \wedge^{i} \tilde{f}_{l}(z)$. On the other hand, $\tilde{f}_{j}(y) \wedge^{i} \widetilde{f}_{j}(z)<\tilde{r}$ for all $j \in I$, which is a contradiction. Thus $\bigvee_{j \in I}^{i}\left(\tilde{f}_{j}(y) \wedge^{i} \tilde{f}_{j}(z)\right)=\left(\bigvee_{j \in I}^{i} \tilde{f}_{j}(y)\right) \wedge^{i}\left(\bigvee_{j \in I}^{i} \tilde{f}_{j}(z)\right)$.

Furthermore, if $x \leq y$, then $\tilde{f}(x) \vee^{i}\left[\left(1-k^{-}\right) / 2,(1-\right.$ $\left.\left.k^{+}\right) / 2\right] \geq \tilde{f}(y)$. Indeed, since $\tilde{f}_{j}$ is an interval valued $(\bar{\epsilon}, \bar{\epsilon} \vee$ $\left.\overline{q_{\tilde{k}}}\right)$-fuzzy quasi-ideal of $S$ for all $j \in I$, we have $\widetilde{f}_{j}(x) \vee^{i}[(1-$ $\left.\left.k^{-}\right) / 2,\left(1-k^{+}\right) / 2\right] \geq \tilde{f}_{j}(y)$ for any $j \in I$. Thus

$$
\begin{aligned}
\tilde{f} & (x) \vee^{i}\left[\frac{1-k^{-}}{2}, \frac{1-k^{+}}{2}\right] \\
& =\left(\bigcup_{j \in I} \widetilde{f}_{j}\right)(x) \vee^{i}\left[\frac{1-k^{-}}{2}, \frac{1-k^{+}}{2}\right]
\end{aligned}
$$




$$
\begin{aligned}
& =\bigvee_{j \in I}^{i}\left(\tilde{f}_{j}(x)\right) \vee^{i}\left[\frac{1-k^{-}}{2}, \frac{1-k^{+}}{2}\right] \\
& =\bigvee_{j \in I}^{i}\left(\tilde{f}_{j}(x) \vee^{i}\left[\frac{1-k^{-}}{2}, \frac{1-k^{+}}{2}\right]\right) \\
& \geq \bigvee_{j \in I}^{i}\left(\tilde{f}_{j}(y)\right)=\left(\bigcup_{j \in I} \tilde{f}_{j}\right)(y)=\tilde{f}(y) .
\end{aligned}
$$

Therefore, $\tilde{f}$ is an interval valued $\left(\bar{\epsilon}, \bar{\epsilon} \vee \overline{q_{\tilde{k}}}\right)$-fuzzy quasi-ideal of $S$ by Theorem 11 .

\section{Prime Interval Valued $\left(\bar{\epsilon}, \bar{\epsilon} \vee \overline{q_{\bar{k}}}\right)$-Fuzzy Quasi-Ideals of Ordered Semigroups}

In this section, we study mainly the prime interval valued $\left(\bar{\epsilon}, \bar{\epsilon} \vee \overline{q_{\tilde{k}}}\right)$-fuzzy quasi-ideals of ordered semigroups and discuss their related properties.

Definition 23. An interval valued $\left(\bar{\epsilon}, \bar{\epsilon} \vee \overline{q_{\tilde{k}}}\right)$-fuzzy quasi-ideal $\tilde{f}$ of an ordered semigroup $S$ is called prime if for all $x, y \in S$ and $\tilde{t} \in D(0,1]$,

$$
x_{\tilde{t}} \bar{\epsilon} \tilde{f}, y_{\tilde{t}} \bar{\epsilon} \widetilde{f} \Longrightarrow(x y)_{\tilde{t}} \bar{\epsilon} \vee \overline{q_{\tilde{k}}} \tilde{f} .
$$

Example 24. Consider the ordered semigroup $S$ given in Example 8 and define an interval valued fuzzy subset $\widetilde{f}$ of $S$ by $\tilde{f}(a)=[0.3,0.4], \tilde{f}(b)=\widetilde{f}(d)=[0.2,0.3]$, and $\tilde{f}(c)=\tilde{f}(e)=$ $[0.1,0.2]$. By routine calculations, $\tilde{f}$ is a prime interval valued $\left(\bar{\epsilon}, \bar{\epsilon} \vee \overline{q_{\tilde{k}}}\right)$-fuzzy quasi-ideal of $S$ for any $\tilde{k} \in E D[0,0.2)$.

Theorem 25. Let $S$ be an ordered semigroup and $\tilde{f}$ an interval valued $\left(\bar{\epsilon}, \bar{\epsilon} \vee \overline{q_{\widetilde{k}}}\right)$-fuzzy quasi-ideal of S. Then $\tilde{f}$ is prime if and only if $\tilde{f}$ satisfies

$$
\tilde{f}(x) \vee^{i} \tilde{f}(y) \vee^{i}\left[\frac{1-k^{-}}{2}, \frac{1-k^{+}}{2}\right] \geq \tilde{f}(x y)
$$

for all $x, y \in S$.

Proof. Let $\tilde{f}$ be a prime interval valued $\left(\bar{\epsilon}, \bar{\epsilon} \vee \overline{q_{\tilde{k}}}\right)$-fuzzy quasiideal of $S$ and $x, y \in S$. Then $\tilde{f}(x) \vee^{i} \tilde{f}(y) \vee^{i}\left[\left(1-k^{-}\right) / 2,(1-\right.$ $\left.\left.k^{+}\right) / 2\right] \geq \widetilde{f}(x y)$. Indeed, if $\widetilde{f}(x) \vee^{i} \widetilde{f}(y) \vee^{i}\left[\left(1-k^{-}\right) / 2,(1-\right.$ $\left.\left.k^{+}\right) / 2\right]<\widetilde{f}(x y)$ for some $x, y \in S$, then there exists $\tilde{t} \in$ $D((1-k) / 2,1]$ such that $\tilde{f}(x) \vee^{i} \tilde{f}(y) \vee^{i}\left[\left(1-k^{-}\right) / 2,\left(1-k^{+}\right) / 2\right]<$ $\tilde{t} \leq \tilde{f}(x y)$. Then $x_{\tilde{t}}, y_{\tilde{t}} \bar{\epsilon} \tilde{f}$, but $\tilde{f}(x y) \geq \tilde{t}, \tilde{f}(x y)+\tilde{t}+$ $\tilde{k}>\left[\left(1-k^{-}\right) / 2,\left(1-k^{+}\right) / 2\right]+\left[\left(1-k^{-}\right) / 2,\left(1-k^{+}\right) / 2\right]+$ $\widetilde{k}=[1,1]$, so $(x y)_{\tilde{t}} \overline{\bar{\epsilon} \vee \overline{q_{\tilde{k}}}} \widetilde{f}$, which is a contradiction. Hence $\widetilde{f}(x) \vee^{i} \tilde{f}(y) \vee^{i}\left[\left(1-k^{-}\right) / 2,\left(1-k^{+}\right) / 2\right] \geq \widetilde{f}(x y)$ for all $x, y \in S$.

Conversely, assume that $\tilde{f}(x) \vee^{i} \tilde{f}(y) \vee^{i}\left[\left(1-k^{-}\right) / 2,(1-\right.$ $\left.\left.k^{+}\right) / 2\right] \geq \tilde{f}(x y)$ for all $x, y \in S$. Let $\widetilde{t} \in D(0,1]$ be such that $x_{\tilde{t}}, y_{\bar{t}} \bar{\epsilon} \widetilde{f}$. Then $\tilde{f}(x)<\widetilde{t}$ and $\widetilde{f}(y)<\widetilde{t}$. Now we consider the following two cases.

Case 1. If $\tilde{t}>\left[\left(1-k^{-}\right) / 2,\left(1-k^{+}\right) / 2\right]$, then, since $\tilde{f}$ is comparable, by hypothesis, we have $\tilde{f}(x y) \leq \tilde{f}(x) \vee^{i} \tilde{f}(y) \vee^{i}\left[\left(1-k^{-}\right) / 2\right.$, $\left.\left(1-k^{+}\right) / 2\right]<\tilde{t} \vee^{i}\left[\left(1-k^{-}\right) / 2,\left(1-k^{+}\right) / 2\right]=\tilde{t}$, that is, $(x y)_{\tilde{t}} \bar{\epsilon} \tilde{f}$. Thus, $(x y)_{\tilde{t}} \bar{\epsilon} \vee \overline{q_{\tilde{k}}} \widetilde{f}$.

Case 2. Let $\tilde{t} \leq\left[\left(1-k^{-}\right) / 2,\left(1-k^{+}\right) / 2\right]$. Then we have $\tilde{f}(x y) \leq$ $\tilde{f}(x) \vee^{i} \tilde{f}(y) \vee^{i}\left[\left(1-k^{-}\right) / 2,\left(1-k^{+}\right) / 2\right] \leq \widetilde{t} \vee^{i}\left[\left(1-k^{-}\right) / 2,(1-\right.$ $\left.\left.k^{+}\right) / 2\right]=\left[\left(1-k^{-}\right) / 2,\left(1-k^{+}\right) / 2\right]$. Hence $\tilde{f}(x)+\widetilde{t}+\widetilde{k} \leq[(1-$ $\left.\left.k^{-}\right) / 2,\left(1-k^{+}\right) / 2\right]+\left[\left(1-k^{-}\right) / 2,\left(1-k^{+}\right) / 2\right]+\widetilde{k}=[1,1]$, that is, $(x y)_{\tilde{t}} \overline{q_{\tilde{k}}} \widetilde{f}$. Therefore, $(x y)_{\tilde{t}} \bar{\epsilon} \vee \overline{q_{\tilde{k}}} \tilde{f}$.

This proves that $\tilde{f}$ is prime.

Remark 26. The hypothesis that the interval valued fuzzy subset $\tilde{f}$ is comparable cannot be removed in the above theorem. Otherwise, the sufficiency of Theorem 25 is not true. We can illustrate it by the following example.

Example 27. We consider the ordered semigroup $S$ := $\{a, b, c, d\}$ defined by the following multiplication "." and the order " $\leq$ ":

$$
\begin{aligned}
& \begin{array}{l|llll}
\cdot & a & b & c & d \\
\hline a & b & b & d & d \\
b & b & b & d & d \\
c & d & d & c & d \\
d & d & d & d & d
\end{array} \\
& \leq:=\{(a, a),(a, b),(b, b),(c, c),(d, b),(d, c),(d, d)\} .
\end{aligned}
$$

Let $\widetilde{f}$ be an interval valued fuzzy subset of $S$ such that $\tilde{f}(a)=\tilde{f}(b)=[0.32,0.32], \tilde{f}(c)=[0.3,0.34]$, and $\tilde{f}(d)=$ $[0.32,0.34]$. By routine calculations, $\tilde{f}$ is an interval valued $\left(\bar{\epsilon}, \bar{\epsilon} \vee \overline{q_{[0.36,0.36]}}\right)$-fuzzy quasi-ideal of $S$, and $\tilde{f}$ satisfies

$$
\tilde{f}(x) \vee^{i} \tilde{f}(y) \vee^{i}\left[\frac{1-0.36}{2}, \frac{1-0.36}{2}\right] \geq \tilde{f}(x y)
$$

for all $x, y \in S$. But $\tilde{f}$ is not prime, since $b_{[0.32,0.34]} \bar{\epsilon} \tilde{f}$ and $c_{[0.32,0.34]} \bar{\epsilon} \tilde{f}$, while

$$
(b c)_{[0.32,0.34]}=d_{[0.32,0.34]} \overline{\bar{\epsilon} \vee \overline{q_{[0.36,0.36]}} \tilde{f} . ~}
$$

Now we will characterize the prime interval valued $(\bar{\epsilon}, \bar{\epsilon} \vee$ $\overline{q_{\widetilde{k}}}$ )-fuzzy quasi-ideals of ordered semigroups by using their level subsets.

Theorem 28. Let $S$ be an ordered semigroup and $\tilde{f}$ an interval valued fuzzy subset of $S$. Then $\tilde{f}$ is a prime interval valued $\left(\bar{\epsilon}, \bar{\epsilon} \vee \overline{q_{\tilde{k}}}\right)$-fuzzy quasi-ideal of $S$ if and only if the level subset $\tilde{f}_{\tilde{t}}$ of $\tilde{f}$ is a prime quasi-ideal of $S$ for all $\tilde{t} \in D((1-k) / 2,1]$ with $\widetilde{f}_{\tilde{t}} \neq \emptyset$.

Proof. Let $\tilde{f}$ be a prime interval valued $\left(\bar{\epsilon}, \bar{\epsilon} \vee \overline{q_{\tilde{k}}}\right)$-fuzzy quasiideal of $S$ and $\tilde{t} \in D((1-k) / 2,1]$. Then, by Theorem 17, $\tilde{f}_{\tilde{t}}(\neq \emptyset)$ is a quasi-ideal of $S$ for all $\tilde{t} \in D((1-k) / 2,1]$. To prove that $\widetilde{f}_{\tilde{t}}$ is prime, let $x y \in \widetilde{f}_{\tilde{t}}$. By Theorem 25 , we have

$$
\tilde{f}(x) \vee^{i} \tilde{f}(y) \vee^{i}\left[\frac{1-k^{-}}{2}, \frac{1-k^{+}}{2}\right] \geq \tilde{f}(x y) \geq \widetilde{t} .
$$


Note that $\tilde{t} \in D((1-k) / 2,1]$ and $\tilde{f}$ satisfies the condition (C). Then $\tilde{f}(x) \vee^{i} \widetilde{f}(y) \geq \widetilde{t}$. Since $\widetilde{f}$ is comparable, we have $\widetilde{f}(x) \geq \widetilde{t}$ or $\widetilde{f}(y) \geq \widetilde{t}$. Thus, $x \in \widetilde{f}_{\tilde{t}}$ or $y \in \tilde{f}_{\tilde{t}}$. This shows that $\tilde{f}_{\tilde{t}}$ is a prime quasi-ideal of $S$ for all $\tilde{t} \in D((1-k) / 2,1]$ with $\tilde{f}_{\tilde{t}} \neq \emptyset$.

Conversely, assume that for every $\widetilde{t} \in D((1-k) / 2,1]$ such that $\widetilde{f}_{\tilde{t}} \neq \emptyset$ the set $\widetilde{f}_{\tilde{t}}$ is a prime quasi-ideal of $S$. Then, by Theorem 17, $\tilde{f}$ is an interval valued $\left(\bar{\epsilon}, \bar{\epsilon} \vee \overline{q_{\tilde{k}}}\right)$-fuzzy quasiideal of $S$. Let $x_{\tilde{t}} \bar{\epsilon} \tilde{f}$ and $x_{\tilde{t}} \bar{\epsilon} \tilde{f}$. Then $x, y \notin \tilde{f}_{\tilde{t}}$. Since $\tilde{f}_{\tilde{t}}$ is prime, we have $x y \notin \tilde{f}_{\tilde{t}}$; that is, $(x y)_{\bar{t}} \bar{\epsilon} \tilde{f}$. Thus, $(x y)_{\tilde{t}} \bar{\epsilon} \vee \overline{q_{\tilde{k}}} \tilde{f}$. Therefore, $\tilde{f}$ is a prime interval valued $\left(\bar{\epsilon}, \bar{\epsilon} \vee \overline{q_{\tilde{k}}}\right)$-fuzzy quasiideal of $S$.

\section{Characterizations of Some Types of Ordered Semigroups}

Throughout the remaining paper, let $Z^{+}$denote the set of all positive integers. In this section we first study the properties of $\tilde{k}$-upper parts of interval valued $\left(\bar{\epsilon}, \bar{\epsilon} \vee \overline{q_{\tilde{k}}}\right)$ fuzzy quasi-ideals of ordered semigroups. Furthermore, we give characterizations of regular, intra-regular, or bi-regular ordered semigroups in terms of interval valued $\left(\bar{\epsilon}, \bar{\epsilon} \vee \overline{q_{\tilde{k}}}\right)$ fuzzy quasi-ideals.

Definition 29. Let $\tilde{f}$ be an interval valued fuzzy subset of an ordered semigroup $S$. Then we define the $\widetilde{k}$-upper part $\tilde{f}_{\widetilde{k}}^{+}$of $\tilde{f}$ as follows:

$$
\tilde{f}_{\tilde{k}}^{+}(x)=\tilde{f}(x) \vee^{i}\left[\frac{1-k^{-}}{2}, \frac{1-k^{+}}{2}\right]
$$

for all $x \in S$.

Clearly, $\tilde{f}_{\tilde{k}}^{+}$is still an interval valued fuzzy subset of $S$. Furthermore, for any $\tilde{f}, \tilde{g} \in I V F(S)$, the interval valued fuzzy subsets $\tilde{f} \cap^{\tilde{k}} \widetilde{g}$ and $\widetilde{f} *^{\tilde{k}} \widetilde{g}$ of $S$ are defined as follows:

$$
\begin{aligned}
& \left(\tilde{f} \cap \cap^{\tilde{g}} \tilde{g}\right)(x)=(\tilde{f} \cap \tilde{g})(x) \vee^{i}\left[\frac{1-k^{-}}{2}, \frac{1-k^{+}}{2}\right], \\
& \left(\tilde{f} *^{\tilde{k}} \tilde{g}\right)(x)=(\tilde{f} * \tilde{g})(x) \vee^{i}\left[\frac{1-k^{-}}{2}, \frac{1-k^{+}}{2}\right],
\end{aligned}
$$

for all $x \in S$.

Lemma 30. Let $S$ be an ordered semigroup and $\tilde{f}, \widetilde{g} \in \operatorname{IVF}(S)$. Then the following statements are true:

(1) $\left(\tilde{f}_{\tilde{k}}^{+}\right)_{\tilde{k}}^{+}=\tilde{f}_{\tilde{k}}^{+}, \tilde{f} \subseteq \tilde{f}_{\tilde{k}}^{+}$.

(2) If $\tilde{f} \subseteq \widetilde{g}$ and $\widetilde{h} \in \operatorname{IVF}(S)$, then $\tilde{f} *^{\tilde{k}} \widetilde{h} \subseteq \tilde{g} * \widetilde{k} \widetilde{h}, \widetilde{h} * \widetilde{k} \widetilde{f} \subseteq$ $\widetilde{h} *{ }^{\widetilde{k}} \tilde{g}$.

(3) $\tilde{f} \cap^{\tilde{k}} \widetilde{g}=\widetilde{f}_{\tilde{k}}^{+} \cap \widetilde{g}_{\widetilde{k}}^{+}$.

(4) $\tilde{f}_{\tilde{k}}^{+} * \tilde{g}_{\tilde{k}}^{+} \subseteq \tilde{f} * \widetilde{k} \widetilde{g}, \widetilde{f}_{\tilde{k}}^{+} * \widetilde{S} \subseteq \tilde{f} * \widetilde{k} \widetilde{S}$, and $\widetilde{S} * \widetilde{f}_{\tilde{k}}^{+} \subseteq$ $\widetilde{S} *{ }^{k} \tilde{f}$. Furthermore, if $A_{x} \neq \emptyset$, then $\left(\tilde{f} *{ }^{\tilde{k}} \tilde{g}\right)(x)=\left(\tilde{f}_{\tilde{k}}^{+} *\right.$

$$
\begin{aligned}
& \left.\tilde{g}_{\tilde{k}}^{+}\right)(x),\left(\tilde{f} *^{\widetilde{k}} \widetilde{S}\right)(x)=\left(\tilde{f}_{\tilde{k}}^{+} * \widetilde{S}\right)(x) \text {, and }\left(\widetilde{S} *^{\tilde{k}} \widetilde{f}\right)(x)= \\
& \left(\widetilde{S} * \widetilde{f}_{\tilde{k}}^{+}\right)(x) .
\end{aligned}
$$

Proof. The proof is straightforward and we omit it.

Lemma 31. Let $A, B$ be any nonempty subsets of an ordered semigroup $S$. Then $\tilde{f}_{A} *^{\tilde{k}} \tilde{f}_{B}=\left(\tilde{f}_{(A B]}\right)_{\tilde{k}}^{+}$, where $\tilde{f}_{A}, \tilde{f}_{B}$, and $\tilde{f}_{(A B]}$ are the interval valued characteristic function of $A, B$, and $(A B]$, respectively.

Proof. Let $x \in S$. If $x \in(A B]$, then $\left(\tilde{f}_{(A B]}\right)_{\tilde{k}}^{+}(x)=[1,1]$ and $x \leq a b$ for some $a \in A$ and $b \in B$. Thus $(a, b) \in A_{x}$, and

$$
\begin{aligned}
& \left(\tilde{f}_{A} * \tilde{k} \tilde{f}_{B}\right)(x) \\
& =\left(\tilde{f}_{A} * \widetilde{f}_{B}\right)(x) \vee^{i}\left[\frac{1-k^{-}}{2}, \frac{1-k^{+}}{2}\right] \\
& =\bigvee_{(y, z) \in A_{x}}^{i}\left[\tilde{f}_{A}(y) \wedge^{i} \tilde{f}_{B}(z)\right] \vee^{i}\left[\frac{1-k^{-}}{2}, \frac{1-k^{+}}{2}\right] \\
& \geq\left(\tilde{f}_{A}(a) \wedge^{i} \tilde{f}_{B}(b)\right) \vee^{i}\left[\frac{1-k^{-}}{2}, \frac{1-k^{+}}{2}\right] \\
& =\left([1,1] \wedge^{i}[1,1]\right) \vee^{i}\left[\frac{1-k^{-}}{2}, \frac{1-k^{+}}{2}\right] \\
& =[1,1]=\left(\tilde{f}_{(A B]}\right)_{\tilde{k}}^{+}(x) .
\end{aligned}
$$

On the other hand, since $\left(\widetilde{f}_{A} * \widetilde{f}_{B}\right)(x) \leq[1,1]$ for all $x \in S$, we have $\left(\tilde{f}_{A} *{ }^{k} \tilde{f}_{B}\right)(x)=\left(\tilde{f}_{A} * \tilde{f}_{B}\right)(x) \vee^{i}\left[\left(1-k^{-}\right) / 2,\left(1-k^{+}\right) / 2\right] \leq$ $[1,1]=\left(\tilde{f}_{(A B]}\right)_{\tilde{k}}^{+}(x)$. Therefore, $\left(\tilde{f}_{A} *^{\widetilde{k}} \tilde{f}_{B}\right)(x)=[1,1]=$ $\left(\tilde{f}_{(A B]}\right)_{\tilde{k}}^{+}(x)$. If $x \notin(A B]$, then $\left(\tilde{f}_{(A B]}\right)_{\tilde{k}}^{-}(x)=\left[\left(1-k^{-}\right) / 2,(1-\right.$ $\left.\left.k^{+}\right) / 2\right]$. We now prove that $\left(\tilde{f}_{A} *^{*} \tilde{f}_{B}\right)(x)=\left[\left(1-k^{-}\right) / 2,(1-\right.$ $\left.\left.k^{+}\right) / 2\right]$. Indeed, if $A_{x}=\emptyset$, then $\left(\widetilde{f}_{A} *^{\tilde{k}} \widetilde{f}_{B}\right)(x)=\left(\tilde{f}_{A} *\right.$ $\left.\tilde{f}_{B}\right)(x) \vee^{i}\left[\left(1-k^{-}\right) / 2,\left(1-k^{+}\right) / 2\right]=[0,0] \vee^{i}\left[\left(1-k^{-}\right) / 2,\left(1-k^{+}\right) / 2\right]$ $=\left[\left(1-k^{-}\right) / 2,\left(1-k^{+}\right) / 2\right]$, and $\left(\widetilde{f}_{A} *^{\widetilde{k}} \widetilde{f}_{B}\right)(x)=\left(\tilde{f}_{(A B]}\right)_{\tilde{k}}^{+}(x)$. If $A_{x} \neq \emptyset$, then $x \leq y z$ for all $(y, z) \in A_{x}$. If $y \in A$ and $z \in B$, then $y z \in A B$, so $x \in(A B]$, which is impossible. Thus $y \notin A$ or $z \notin B$. If $y \notin A$, then $\tilde{f}_{A}(y)=[0,0]$. Since $\tilde{f}_{B}(z) \geq[0,0]$, we have $\tilde{f}_{A}(y) \wedge^{i} \widetilde{f}_{B}(z)=[0,0]$. If $z \notin B$, then, as in the previous case, we have $\widetilde{f}_{A}(y) \wedge^{i} \widetilde{f}_{B}(z)=[0,0]$. Thus we have

$$
\begin{aligned}
& \left(\tilde{f}_{A} *^{*} \widetilde{f}_{B}\right)(x) \\
& \quad=\left(\widetilde{f}_{A} * \widetilde{f}_{B}\right)(x) \vee^{i}\left[\frac{1-k^{-}}{2}, \frac{1-k^{+}}{2}\right] \\
& \quad=\bigvee_{(y, z) \in A_{x}}^{i}\left[\tilde{f}_{A}(y) \wedge^{i} \tilde{f}_{B}(z)\right] \vee^{i}\left[\frac{1-k^{-}}{2}, \frac{1-k^{+}}{2}\right] \\
& \quad=\left[\frac{1-k^{-}}{2}, \frac{1-k^{+}}{2}\right] .
\end{aligned}
$$

This completes the proof. 
In Example 13, we have shown that an interval valued $\left(\bar{\epsilon}, \bar{\epsilon} \vee \overline{q_{\tilde{k}}}\right)$-fuzzy quasi-ideal of an ordered semigroup $S$ is not necessarily an interval valued fuzzy quasi-ideal of $S$. In the following theorem, we show that if $\widetilde{f}$ is an interval valued $\left(\bar{\epsilon}, \bar{\epsilon} \vee \overline{q_{\tilde{k}}}\right)$-fuzzy quasi-ideal of $S$, then the $\widetilde{k}$-upper part $\widetilde{f}_{\widetilde{k}}^{+}$ of $\tilde{f}$ is an interval valued fuzzy quasi-ideal of $S$.

Theorem 32. Let $S$ be an ordered semigroup and $\tilde{f}$ an interval valued $\left(\bar{\epsilon}, \bar{\epsilon} \vee \overline{q_{\widetilde{k}}}\right)$-fuzzy quasi-ideal of $S$. Then the $\widetilde{k}$-upper part $\tilde{f}_{\tilde{k}}^{+}$of $\tilde{f}$ is an interval valued fuzzy quasi-ideal of $S$.

Proof. Let $\tilde{f}$ be an interval valued $\left(\bar{\epsilon}, \bar{\epsilon} \vee \overline{q_{\tilde{k}}}\right)$-fuzzy quasi-ideal of $S$ and $x, y \in S$. Then, by Theorem $10, \widetilde{f}(x) \vee^{i}\left[\left(1-k^{-}\right) / 2,(1-\right.$ $\left.\left.k^{+}\right) / 2\right] \geq(\widetilde{f} * \widetilde{S})(x) \wedge^{i}(\widetilde{S} * \widetilde{f})(x)$, and we have

$$
\begin{aligned}
\tilde{f}_{\tilde{k}}^{+}(x)= & \tilde{f}(x) \vee^{i}\left[\frac{1-k^{-}}{2}, \frac{1-k^{+}}{2}\right] \\
\geq & \left((\widetilde{f} * \widetilde{S})(x) \vee^{i}\left[\frac{1-k^{-}}{2}, \frac{1-k^{+}}{2}\right]\right) \\
& \wedge^{i}\left((\widetilde{S} * \widetilde{f})(x) \vee^{i}\left[\frac{1-k^{-}}{2}, \frac{1-k^{+}}{2}\right]\right) \\
= & (\widetilde{f} * \widetilde{k} \widetilde{S})(x) \wedge^{i}\left(\widetilde{S}^{*} \widetilde{f} \tilde{f}\right)(x) \\
\geq & \left(\widetilde{f}_{\tilde{k}}^{+} * \widetilde{S}\right)(x) \wedge^{i}\left(\widetilde{S} * \widetilde{f}_{\tilde{k}}^{+}\right)(x) .
\end{aligned}
$$

It implies that $\left(\widetilde{f}_{\tilde{k}}^{+} * \widetilde{S}\right) \cap\left(\widetilde{S} * \widetilde{f}_{\widetilde{k}}^{+}\right) \subseteq \widetilde{f}_{\widetilde{k}}^{+}$. Moreover, if $x \leq y$, then $\widetilde{f}_{\tilde{k}}^{+}(x) \geq \widetilde{f}_{\tilde{k}}^{+}(y)$. Indeed, since $\tilde{f}$ is an interval valued $(\bar{\epsilon}, \bar{\epsilon} \vee$ $\left.\overline{q_{\tilde{k}}}\right)$-fuzzy quasi-ideal of $S$, we have $\widetilde{f}(x) \vee^{i}\left[\left(1-k^{-}\right) / 2,(1-\right.$ $\left.\left.k^{+}\right) / 2\right] \geq \tilde{f}(y)$, and so $\tilde{f}_{\tilde{k}}^{+}(x)=\tilde{f}(x) \vee^{i}\left[\left(1-k^{-}\right) / 2,\left(1-k^{+}\right) / 2\right]$ $=\left(\tilde{f}(x) \vee^{i}\left[\left(1-k^{-}\right) / 2,\left(1-k^{+}\right) / 2\right]\right) \vee^{i}\left[\left(1-k^{-}\right) / 2,\left(1-k^{+}\right) / 2\right] \geq$ $\widetilde{f}(y) \vee^{i}\left[\left(1-k^{-}\right) / 2,\left(1-k^{+}\right) / 2\right]=\widetilde{f}_{\widetilde{k}}^{+}(y)$. Therefore, $\widetilde{f}_{\widetilde{k}}^{+}$is an interval valued fuzzy quasi-ideal of $S$.

Theorem 33. Let $S$ be an ordered semigroup and $\tilde{f}$ an interval valued fuzzy subset of $S$. Then $\tilde{f}$ is an interval valued $(\bar{\epsilon}, \bar{\epsilon} \vee$ $\left.\overline{q_{\tilde{k}}}\right)$-fuzzy quasi-ideal of $S$ if and only if $\tilde{f}$ satisfies the following assertions:

(1) $x \leq y \Rightarrow \tilde{f}(x) \vee^{i}\left[\left(1-k^{-}\right) / 2,\left(1-k^{+}\right) / 2\right] \geq \tilde{f}(y)$ for all $x, y \in S$.

(2) $(\widetilde{f} * \widetilde{k} \widetilde{S}) \cap\left(\widetilde{S} *^{\widetilde{k}} \widetilde{f}\right) \subseteq \widetilde{f}_{\widetilde{k}}^{+}$

Proof. It is obvious by Lemma 30 and Theorem 10.

Lemma 34 (cf. [37]). An ordered semigroup $S$ is regular and intra-regular if and only if $Q=\left(Q^{2}\right]$ for every quasi-ideal $Q$ of $S$.

Now we give characterizations of an ordered semigroup which is both regular and intra-regular by interval valued $\left(\bar{\epsilon}, \bar{\epsilon} \vee \overline{q_{\tilde{k}}}\right)$-fuzzy quasi-ideals.
Theorem 35. Let $S$ be an ordered semigroup. Then the following conditions are equivalent:

(1) $S$ is regular and intra-regular.

(2) $\tilde{f} * \widetilde{k} \tilde{f}=\widetilde{f}_{\tilde{k}}^{+}$for every interval valued $\left(\bar{\epsilon}, \bar{\epsilon} \vee \overline{q_{\tilde{k}}}\right)$-fuzzy quasi-ideal $\tilde{f}$ of $S$.

(3) $\tilde{f} \cap^{\tilde{k}} \tilde{g} \subseteq(\tilde{f} * \widetilde{k} \tilde{g}) \cap(\widetilde{g} * \tilde{k} \tilde{f})$ for any interval valued $(\bar{\epsilon}, \bar{\epsilon} \mathrm{V}$ $\left.\overline{q_{\tilde{k}}}\right)$-fuzzy quasi-ideals $\widetilde{f}$ and $\widetilde{g}$ of $S$.

Proof. (1) $\Rightarrow$ (3). Let $\tilde{f}$ and $\tilde{g}$ be interval valued $\left(\bar{\epsilon}, \bar{\epsilon} \vee \overline{q_{\tilde{k}}}\right)$-fuzzy quasi-ideals of $S$ and $a \in S$. Then, since $S$ is both regular and intra-regular, there exists $x \in S$ such that $a \leq a \times a(\leq a \times a \times a)$, and there exist $y, z \in S$ such that $a \leq y a^{2} z$. Thus

$$
a \leq a x a \leq \operatorname{axaxa} \leq a x\left(y a^{2} z\right) x a=(a x y a)(a z x a),
$$

that is, $(a x y a, a z x a) \in A_{a}$. Since $\tilde{f}$ is an interval valued $\left(\bar{\epsilon}, \bar{\epsilon} \vee \overline{q_{\tilde{k}}}\right)$-fuzzy quasi-ideal of $S$ and axya $\leq a(x y a)$, axya $\leq$ $(a x y) a$, by Theorem 11, we have

$$
\begin{aligned}
\tilde{f}_{\tilde{k}}^{+} & (\text {axya }) \\
& =\tilde{f}(\text { axya }) \vee^{i}\left[\frac{1-k^{-}}{2}, \frac{1-k^{+}}{2}\right] \\
& =\left(\tilde{f}(\text { axya }) \vee^{i}\left[\frac{1-k^{-}}{2}, \frac{1-k^{+}}{2}\right]\right) \vee^{i}\left[\frac{1-k^{-}}{2}, \frac{1-k^{+}}{2}\right] \\
& \geq\left(\tilde{f}(a) \wedge^{i} \tilde{f}(a)\right) \vee^{i}\left[\frac{1-k^{-}}{2}, \frac{1-k^{+}}{2}\right] \\
& =\tilde{f}(a) \vee^{i}\left[\frac{1-k^{-}}{2}, \frac{1-k^{+}}{2}\right]=\widetilde{f}_{\tilde{k}}^{+}(a) .
\end{aligned}
$$

By azxa $\leq a(z x a), a z x a \leq(a z x) a$, in a similar way we can get $\widetilde{g}_{\widetilde{k}}^{+}(a z x a) \geq \widetilde{g}_{\widetilde{k}}^{+}(a)$. Thus we have

$$
\begin{aligned}
\left(\tilde{f} *^{\tilde{k}} \tilde{g}\right)(a) & =\left(\tilde{f}_{\tilde{k}}^{+} * \tilde{g}_{\tilde{k}}^{+}\right)(a) \\
& =\bigvee_{(p, q) \in A_{a}}^{i}\left[\tilde{f}_{\tilde{k}}^{+}(p) \wedge^{i} \tilde{g}_{\tilde{k}}^{+}(q)\right] \\
& \geq \widetilde{f}_{\tilde{k}}^{+}(a x y a) \wedge^{i} \widetilde{g}_{\tilde{k}}^{+}(a z x a) \\
& \geq \tilde{f}_{\tilde{k}}^{+}(a) \wedge^{i} \widetilde{g}_{\tilde{k}}^{+}(a)=\left(\tilde{f}_{\tilde{k}}^{+} \cap \tilde{g}_{\tilde{k}}^{+}\right)(a) \\
& =(\tilde{f} \cap \tilde{k} \tilde{g})(a),
\end{aligned}
$$

which means that $\tilde{f} \cap \tilde{k} \widetilde{g} \subseteq \tilde{f} * \tilde{k} \widetilde{g}$. In the same way, we can show that $\tilde{f} \cap^{\tilde{k}} \tilde{g} \subseteq \tilde{g} *^{\tilde{k}} \widetilde{f}$. Hence, $\tilde{f} \cap^{\tilde{k}} \tilde{g} \subseteq\left(\tilde{f} *^{\tilde{k}} \widetilde{g}\right) \cap\left(\widetilde{g} *{ }^{\tilde{k}} \widetilde{f}\right)$.

(3) $\Rightarrow(1)$. Take $\widetilde{f}=\widetilde{g}$ in (3) and we get $\widetilde{f} *^{\widetilde{k}} \widetilde{f} \supseteq \widetilde{f} \cap^{\widetilde{k}} \widetilde{f}=$ $\tilde{f}_{\tilde{k}}^{+} \cap \tilde{f}_{\tilde{k}}^{+}=\tilde{f}_{\widetilde{k}}^{+}$. On the other hand, by Lemma 30(2) and Theorem 33, we have $\tilde{f} * \widetilde{k} \widetilde{f} \subseteq(\widetilde{f} * \widetilde{k} \widetilde{S}) \cap(\widetilde{S} * \widetilde{k} \widetilde{f}) \subseteq \widetilde{f}_{\widetilde{k}}^{+}$. Thus we deduce that $\tilde{f} * \tilde{k} \tilde{f}=\tilde{f}_{\tilde{k}}^{+}$. 
(2) $\Rightarrow(1)$. Let $Q$ be a quasi-ideal of $S$. By Lemma 19, the interval valued characteristic function $\widetilde{f}_{Q}$ of $Q$ is an interval valued $\left(\bar{\epsilon}, \bar{\epsilon} \vee \overline{q_{\tilde{k}}}\right)$-fuzzy quasi-ideal of $S$. Then, by hypothesis and Lemma 31, we have

$$
\left(\tilde{f}_{\left(Q^{2}\right]}\right)_{\tilde{k}}^{+}=\tilde{f}_{\mathrm{Q}} *^{\tilde{k}} \tilde{f}_{\mathrm{Q}}=\left(\tilde{f}_{\mathrm{Q}}\right)_{\tilde{k}}^{+},
$$

from which we deduce that $\left(Q^{2}\right]=Q$. By Lemma 34, $S$ is regular and intra-regular.

An ordered semigroup $(S, \cdot, \leq)$ is called left (resp., right) regular if for each $a \in S$ there exists $x \in S$ such that $a \leq x a^{2}$ (resp., $a \leq a^{2} x$ ), that is, $a \in\left(S a^{2}\right]$ (resp., $a \in\left(a^{2} S\right]$ ) (see [24]). An ordered semigroup $(S, \cdot, \leq)$ is called bi-regular if it is both left regular and right regular. Clearly, an ordered semigroup $S$ is bi-regular if and only if $a \in\left(S a^{2}\right] \cap\left(a^{2} S\right]$ for every $a \in S$.

Definition 36. Let $S$ be an ordered semigroup and $\tilde{f}$ an interval valued $\left(\bar{\epsilon}, \bar{\epsilon} \vee \overline{q_{\tilde{k}}}\right)$-fuzzy quasi-ideal of $S$. Then $\tilde{f}$ is called completely semiprime if $\tilde{f}_{\tilde{k}}^{+}(a) \geq \widetilde{f}\left(a^{2}\right)$ for any $a \in S$.

Now we will give some characterizations of bi-regular ordered semigroups in terms of interval valued $\left(\bar{\epsilon}, \bar{\epsilon} \vee \overline{q_{\bar{k}}}\right)$ fuzzy quasi-ideals.

Theorem 37. Let $S$ be an ordered semigroup. Then the following statements are equivalent:

(1) $S$ is bi-regular.

(2) For each interval valued $\left(\bar{\epsilon}, \bar{\epsilon} \vee \overline{q_{\tilde{k}}}\right)$-fuzzy quasi-ideal $\tilde{f}$ of $S, \tilde{f}_{\tilde{k}}^{+}(a)=\tilde{f}_{\tilde{k}}^{+}\left(a^{2}\right)$ for any $a \in S$.

(3) For each interval valued $\left(\bar{\epsilon}, \bar{\epsilon} \vee \overline{q_{\tilde{k}}}\right)$-fuzzy quasi-ideal $\tilde{f}$ of $S, \widetilde{f}_{\tilde{k}}^{+}\left(a^{n}\right)=\tilde{f}_{\widetilde{k}}^{+}\left(a^{n+1}\right)$ for any $a \in S, n \in Z^{+}$.

(4) Every interval valued $\left(\bar{\epsilon}, \bar{\epsilon} \vee \overline{q_{\tilde{k}}}\right)$-fuzzy quasi-ideal $\tilde{f}$ of $S$ is completely semiprime.

Proof. (1) $\Rightarrow(2)$. Let $S$ be a bi-regular ordered semigroup and $\tilde{f}$ an interval valued $\left(\bar{\epsilon}, \bar{\epsilon} \vee \overline{q_{\tilde{k}}}\right)$-fuzzy quasi-ideal of $S$ and let $a \in S$. Since $S$ is bi-regular, we have $a \in\left(a^{2} S\right]$ and $a \in\left(S a^{2}\right]$. Then there exist $x, y \in S$ such that $a \leq a^{2} x$ and $a \leq y a^{2}$. Then, by Theorem 11, we have

$$
\begin{aligned}
\tilde{f}_{\tilde{k}}^{+}(a) & =\tilde{f}(a) \vee^{i}\left[\frac{1-k^{-}}{2}, \frac{1-k^{+}}{2}\right] \\
& =\left(\tilde{f}(a) \vee^{i}\left[\frac{1-k^{-}}{2}, \frac{1-k^{+}}{2}\right]\right) \vee^{i}\left[\frac{1-k^{-}}{2}, \frac{1-k^{+}}{2}\right] \\
& \geq\left(\tilde{f}\left(a^{2}\right) \wedge^{i} \tilde{f}\left(a^{2}\right)\right) \vee^{i}\left[\frac{1-k^{-}}{2}, \frac{1-k^{+}}{2}\right] \\
& =\tilde{f}\left(a^{2}\right) \vee^{i}\left[\frac{1-k^{-}}{2}, \frac{1-k^{+}}{2}\right]=\tilde{f}_{\tilde{k}}^{+}\left(a^{2}\right) .
\end{aligned}
$$

On the other hand, since $a^{2} \leq a a$ and $a^{2} \leq a a$, similar to the above proof, we can show that $\widetilde{f}_{\tilde{k}}^{+}\left(a^{2}\right) \geq \widetilde{f}_{\tilde{k}}^{+}(a)$. Therefore, $\tilde{f}_{\tilde{k}}^{+}(a)=\widetilde{f}_{\tilde{k}}^{+}\left(a^{2}\right)$ for any $a \in S$.
(2) $\Rightarrow(3)$. Let $a \in S$ and $n \in Z^{+}$. Then, by Lemma 30(4), we have

$$
\begin{aligned}
\left(\tilde{f} *^{\widetilde{k}} \widetilde{S}\right)\left(a^{4 n}\right) & =\left(\tilde{f}_{\tilde{k}}^{+} * \widetilde{S}\right)\left(a^{4 n}\right) \\
& =\bigvee_{(x, y) \in A_{a^{4 n}}}^{i}\left[\widetilde{f}_{\tilde{k}}^{+}(x) \wedge^{i} \widetilde{S}(y)\right] \\
& \geq \widetilde{f}_{\tilde{k}}^{+}\left(a^{n+1}\right) \wedge^{i} \widetilde{S}\left(a^{3 n-1}\right) \\
& =\widetilde{f}_{\widetilde{k}}^{+}\left(a^{n+1}\right) \wedge^{i}[1,1]=\tilde{f}_{\tilde{k}}^{+}\left(a^{n+1}\right) .
\end{aligned}
$$

Similarly, it can be shown that $\left(\widetilde{S} *^{\widetilde{k}} \widetilde{f}\right)\left(a^{4 n}\right) \geq \widetilde{f}_{\widetilde{k}}^{+}\left(a^{n+1}\right)$. Thus, by hypothesis and Theorem 33, we have

$$
\begin{aligned}
\tilde{f}_{\tilde{k}}^{+}\left(a^{n}\right) & =\tilde{f}_{\tilde{k}}^{+}\left(a^{2 n}\right)=\widetilde{f}_{\tilde{k}}^{+}\left(a^{4 n}\right) \\
& \geq\left(\left(\widetilde{f} *^{\tilde{k}} \widetilde{S}\right) \cap\left(\widetilde{S} *^{\widetilde{k}} \widetilde{f}\right)\right)\left(a^{4 n}\right) \\
& =\left(\widetilde{f} *^{\tilde{k}} \widetilde{S}\right)\left(a^{4 n}\right) \wedge^{i}\left(\widetilde{S} *^{\tilde{k}} \widetilde{f}\right)\left(a^{4 n}\right) \\
& \geq \widetilde{f}_{\tilde{k}}^{+}\left(a^{n+1}\right) \wedge^{i} \widetilde{f}_{\tilde{k}}^{+}\left(a^{n+1}\right)=\tilde{f}_{\tilde{k}}^{+}\left(a^{n+1}\right) .
\end{aligned}
$$

On the other hand, let $a \in S$. Then we have

$$
\begin{aligned}
\left(\tilde{f} *^{\tilde{k}} \widetilde{S}\right)\left(a^{n+1}\right) & =\left(\tilde{f}_{\widetilde{k}}^{+} * \widetilde{S}\right)\left(a^{n+1}\right) \\
& =\bigvee_{(x, y) \in A_{a^{n+1}}}^{i}\left[\tilde{f}_{\tilde{k}}^{+}(x) \wedge^{i} \widetilde{S}(y)\right] \\
& \geq \tilde{f}_{\tilde{k}}^{+}\left(a^{n}\right) \wedge^{i} \widetilde{S}(a) \\
& =\widetilde{f}_{\tilde{k}}^{+}\left(a^{n}\right) \wedge^{i}[1,1]=\tilde{f}_{\tilde{k}}^{+}\left(a^{n}\right),
\end{aligned}
$$

for any $n \in Z^{+}$. Similarly, $\left(\widetilde{S} *^{\widetilde{k}} \widetilde{f}\right)\left(a^{n+1}\right) \geq \widetilde{f}_{\tilde{k}}^{+}\left(a^{n}\right)$. Since $\tilde{f}$ is an interval valued $\left(\bar{\epsilon}, \bar{\epsilon} \vee \overline{q_{\widetilde{k}}}\right)$-fuzzy quasi-ideal of $S$, by hypothesis, we have

$$
\begin{aligned}
\tilde{f}_{\tilde{k}}^{+}\left(a^{n+1}\right) & \geq\left(\left(\tilde{f} *^{\widetilde{k}} \widetilde{S}\right) \cap\left(\widetilde{S} *^{\tilde{k}} \widetilde{f}\right)\right)\left(a^{n+1}\right) \\
& =\left(\widetilde{f} *^{\widetilde{k}} \widetilde{S}\right)\left(a^{n+1}\right) \wedge^{i}\left(\widetilde{S} *^{\widetilde{k}} \widetilde{f}\right)\left(a^{n+1}\right) \\
& \geq \tilde{f}_{\tilde{k}}^{+}\left(a^{n}\right) \wedge^{i} \widetilde{f}_{\tilde{k}}^{+}\left(a^{n}\right)=\tilde{f}_{\tilde{k}}^{+}\left(a^{n}\right) .
\end{aligned}
$$

(3) $\Rightarrow(2)$ and $(2) \Rightarrow(4)$ are obvious.

$(4) \Rightarrow(1)$. Let $a \in S$. We consider the quasi-ideal $Q\left(a^{2}\right)$ of $S$ generated by $a^{2}$. By Lemma 19, the interval valued characteristic function $\widetilde{f}_{\mathrm{Q}\left(a^{2}\right)}$ of $Q\left(a^{2}\right)$ is an interval valued $\left(\bar{\epsilon}, \bar{\epsilon} \vee \overline{q_{\tilde{k}}}\right)$-fuzzy quasi-ideal of $S$. By hypothesis, $\left(\tilde{f}_{\mathrm{Q}\left(a^{2}\right)}\right)_{\tilde{k}}^{+}(a) \geq$

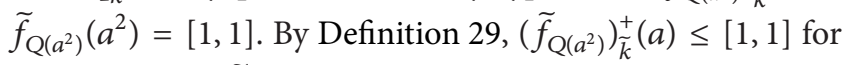
all $a \in S$. Thus $\left(\widetilde{f}_{\mathrm{Q}\left(a^{2}\right)}\right)_{\tilde{k}}^{+}(a)=[1,1]$, and we have

$$
a \in Q\left(a^{2}\right)=\left(a^{2} \cup\left(\left(a^{2} S\right] \cap\left(S a^{2}\right]\right)\right] .
$$


Then $a \leq t$ for some $t \in a^{2} \cup\left(\left(a^{2} S\right] \cap\left(S a^{2}\right]\right)$. If $t=a^{2}$, then $a \leq$ $a^{2}=a a \leq a^{2} a^{2}=a a a^{2} \leq a^{2} a a^{2} \in a^{2} S a^{2} \subseteq\left(a^{2} S\right] \cap\left(S a^{2}\right]$, that is, $a \in\left(a^{2} S\right] \cap\left(S a^{2}\right]$. If $t \in\left(a^{2} S\right] \cap\left(S a^{2}\right]$, then $a \in\left(a^{2} S\right] \cap\left(S a^{2}\right]$. Therefore, $S$ is bi-regular.

\section{Conclusion}

It is well known that ordered semigroups are basic structures in many applied branches like automata and formal languages, coding theory, finite state machines, and others. Due to these possibilities of applications, semigroups and related structures are presently, extensively investigated in fuzzy settings. In particular, generalized fuzzy ordered semigroups play an important role in the mentioned applications. Interval valued fuzzy quasi-ideals of an ordered semigroup with special properties always play an important role in the study of ordered semigroups structure. The interval valued ordered fuzzy points of an ordered semigroup are key to describe the algebraic subsystems of ordered semigroups. In this paper we have introduced the concept of interval valued $\left(\bar{\epsilon}, \bar{\epsilon} \vee \overline{q_{\tilde{k}}}\right)$ fuzzy quasi-ideals and prime (completely semiprime) interval valued $\left(\bar{\epsilon}, \bar{\epsilon} \vee \overline{q_{\bar{k}}}\right)$-fuzzy quasi-ideals of an ordered semigroup and investigated their related properties. Furthermore, we have given some characterizations of regular, intra-regular, or bi-regular ordered semigroups in terms of interval valued $\left(\bar{\epsilon}, \bar{\epsilon} \vee \overline{q_{\widetilde{k}}}\right)$-fuzzy quasi-ideals. The results obtained in this paper are fundamental for the advanced study on ordered semigroups and (generalized) fuzzy ordered semigroups, which we will present in the next paper. As an application of the results of this paper, the corresponding results of semigroups (without order) are also obtained.

\section{Conflict of Interests}

The authors declare that there is no conflict of interests regarding the publication of this paper.

\section{Acknowledgments}

This work was supported by the Natural Science Foundation of China (no. 11371177, 11271040, and 11361027) and the Anhui Provincial Excellent Youth Talent Foundation (no. 2012SQRL115ZD).

\section{References}

[1] L. A. Zadeh, "Fuzzy sets," Information and Control, vol. 8, pp. 338-353, 1965.

[2] A. Rosenfeld, "Fuzzy groups," Journal of Mathematical Analysis and Applications, vol. 35, pp. 512-517, 1971.

[3] L. A. Zadeh, "The concept of a linguistic variable and its application to approximate reasoning. I," Information Sciences, vol. 8, pp. 199-249, 1975.

[4] R. Biswas, "Rosenfeld's fuzzy subgroups with interval-valued membership functions," Fuzzy Sets and Systems, vol. 63, no. 1, pp. 87-90, 1994.

[5] B. Davvaz, O. Kazanc1, and S. Yamak, "Generalized fuzzy nary subpolygroups endowed with interval valued membership functions," Journal of Intelligent and Fuzzy Systems, vol. 20, pp. 159-168, 2009.

[6] B. Davvaz and V. Leoreanu-Fotea, "Applications of interval valued fuzzy $n$-ary polygroups with respect to $t$-norms ( $t$ conorms)," Computers and Mathematics with Applications, vol. 57, no. 8, pp. 1413-1424, 2009.

[7] B. Davvaz, J. Zhan, and K. P. Shum, "Generalized fuzzy $H_{v^{-}}$ submodules endowed with interval valued membership functions," Information Sciences, vol. 178, no. 15, pp. 3147-3159, 2008.

[8] X. Ma and J. Zhan, "On fuzzy $h$-ideals of hemirings," Journal of Systems Science and Complexity, vol. 20, no. 3, pp. 470-478, 2007.

[9] M. Shabir and I. A. Khan, "Interval-valued fuzzy ideals generated by an interval-valued fuzzy subset in ordered semigroups," Mathware and Soft Computing, vol. 15, no. 3, pp. 263-272, 2008.

[10] S. Yamak, O. Kazanc1, and B. Davvaz, "Applications of interval valued $t$-norms ( $t$-conorms) to fuzzy $n$-ary sub-hypergroups," Information Sciences, vol. 178, no. 20, pp. 3957-3972, 2008.

[11] S. K. Bhakat and P. Das, "On the definition of a fuzzy subgroup," Fuzzy Sets and Systems, vol. 51, no. 2, pp. 235-241, 1992.

[12] S. K. Bhakat and P. Das, “( $\epsilon, \in \vee q)$-fuzzy subgroup," Fuzzy Sets and Systems, vol. 80, no. 3, pp. 359-368, 1996.

[13] P. M. Pu and Y. M. Liu, "Fuzzy topology. I. Neighborhood structure of a fuzzy point and Moore-Smith convergence," Journal of Mathematical Analysis and Applications, vol. 76, no. 2, pp. 571-599, 1980.

[14] B. Davvaz, " $(\epsilon, \epsilon \vee q)$-fuzzy subnearrings and ideals," Soft Computing, vol. 10, no. 3, pp. 206-211, 2006.

[15] Y. B. Jun, "On $(\alpha, \beta)$-fuzzy subalgebras of BCK/BCI-algebras," Bulletin of the Korean Mathematical Society, vol. 42, no. 4, pp. 703-711, 2005.

[16] Y. B. Jun and S. Z. Song, "Generalized fuzzy interior ideals in semigroups," Information Sciences, vol. 176, no. 20, pp. 30793093, 2006.

[17] O. Kazanc1 and S. Yamak, "Generalized fuzzy bi-ideals of semigroup," Soft Computing, vol. 12, no. 11, pp. 1119-1124, 2008.

[18] X. Ma and J. Zhan, "Generalized fuzzy $h$-bi-ideals and $h$-quasiideals of hemirings," Information Sciences, vol. 179, no. 9, pp. 1249-1268, 2009.

[19] M. Shabir, Y. B. Jun, and Y. Nawaz, "Semigroups characterized by $\left(\epsilon, \in \vee q_{k}\right)$-fuzzy ideals," Computers and Mathematics with Applications, vol. 60, no. 5, pp. 1473-1493, 2010.

[20] J. Zhan, B. Davvaz, and K. P. Shum, "A new view of fuzzy hypernear-rings," Information Sciences, vol. 178, no. 2, pp. 425438, 2008.

[21] N. Kehayopulu and M. Tsingelis, "Fuzzy sets in ordered groupoids," Semigroup Forum, vol. 65, no. 1, pp. 128-132, 2002.

[22] Y. B. Jun, A. Khan, and M. Shabir, "Ordered semigroups characterized by their $(\epsilon, \in \vee q)$-fuzzy bi-ideals," Bulletin of the Malaysian Mathematical Sciences Society, vol. 32, no. 3, pp. 391408, 2009.

[23] N. Kehayopulu and M. Tsingelis, "Regular ordered semigroups in terms of fuzzy subsets," Information Sciences, vol. 176, no. 24, pp. 3675-3693, 2006.

[24] N. Kehayopulu and M. Tsingelis, "Left regular and intra-regular ordered semigroups in terms of fuzzy subsets," Quasigroups and Related Systems, vol. 14, no. 2, pp. 167-178, 2006.

[25] N. Kehayopulu and M. Tsingelis, "Fuzzy right, left, quasiideals, bi-ideals in ordered semigroups," Lobachevskii Journal of Mathematics, vol. 30, no. 1, pp. 17-22, 2009. 
[26] A. Khan, Y. B. Jun, and M. Shabir, "A study of generalized fuzzy ideals in ordered semigroups," Neural Computing and Applications, vol. 21, pp. 69-78, 2012.

[27] M. Shabir and A. Khan, "Fuzzy quasi-ideals of ordered semigroups," Bulletin of the Malaysian Mathematical Sciences Society, vol. 34, no. 1, pp. 87-102, 2011.

[28] J. Tang and X. Y. Xie, "Characterizations of regular ordered semigroups by generalized fuzzy ideals," Journal of Intelligent and Fuzzy Systems, vol. 26, no. 1, pp. 239-252, 2014.

[29] X. Y. Xie and J. Tang, "Fuzzy radicals and prime fuzzy ideals of ordered semigroups," Information Sciences, vol. 178, no. 22, pp. 4357-4374, 2008.

[30] X. Y. Xie and J. Tang, "Regular ordered semigroups and intraregular ordered semigroups in terms of fuzzy subsets," Iranian Journal of Fuzzy Systems, vol. 7, no. 2, pp. 121-140, 2010.

[31] A. Khan, Y. B. Jun, and M. Shabir, "Ordered semigroups characterized by interval valued $(\epsilon, \epsilon \vee q)$-fuzzy bi-ideals," Journal of Intelligent and Fuzzy Systems, vol. 25, no. 1, pp. 5768, 2013.

[32] B. Davvaz, A. Khan, N. H. Sarmin, and H. Khan, "More general forms of interval valued fuzzy filters of ordered semigroups," International Journal of Fuzzy Systems, vol. 15, no. 2, pp. 110-126, 2013.

[33] O. Stienfeld, Quasi-Ideals in Rings and Semigroups, Akademiakiado, Budapest, Hungary, 1978.

[34] N. Kehayopulu, "On regular, intra-regular ordered semigroups," Pure Mathematics and Applications, vol. 4, no. 4, pp. 447-461, 1993.

[35] N. Kehayopulu, "On weakly prime ideals of ordered semigroups," Mathematica Japonica, vol. 35, no. 6, pp. 1051-1056, 1990.

[36] N. Kehayopulu and M. Tsingelis, "The embedding of an ordered groupoid into a poe-groupoid in terms of fuzzy sets," Information Sciences, vol. 152, pp. 231-236, 2003.

[37] X. Y. Xie, An Introduction to Ordered Semigroup Theory, Kexue Press, Beijing, China, 2001.

[38] X. Y. Xie and M. F. Wu, The Theory of Fuzzy Semigroups, Kexue Press, Beijing, China, 2005.

[39] N. Kehayopulu, "On regular duo ordered semigroups," Mathematica Japonica, vol. 37, no. 3, pp. 535-540, 1992. 


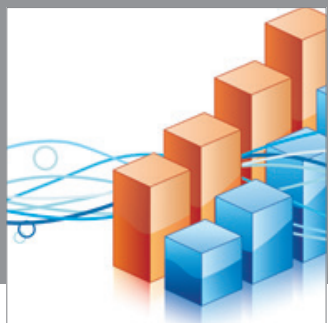

Advances in

Operations Research

mansans

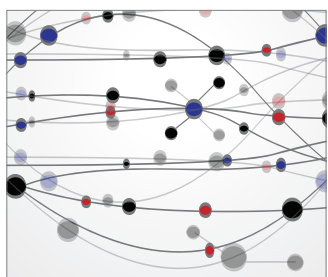

The Scientific World Journal
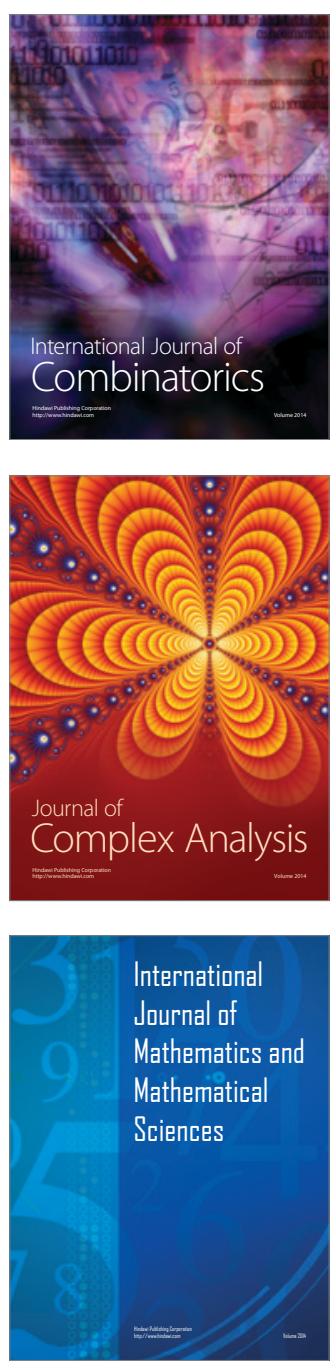
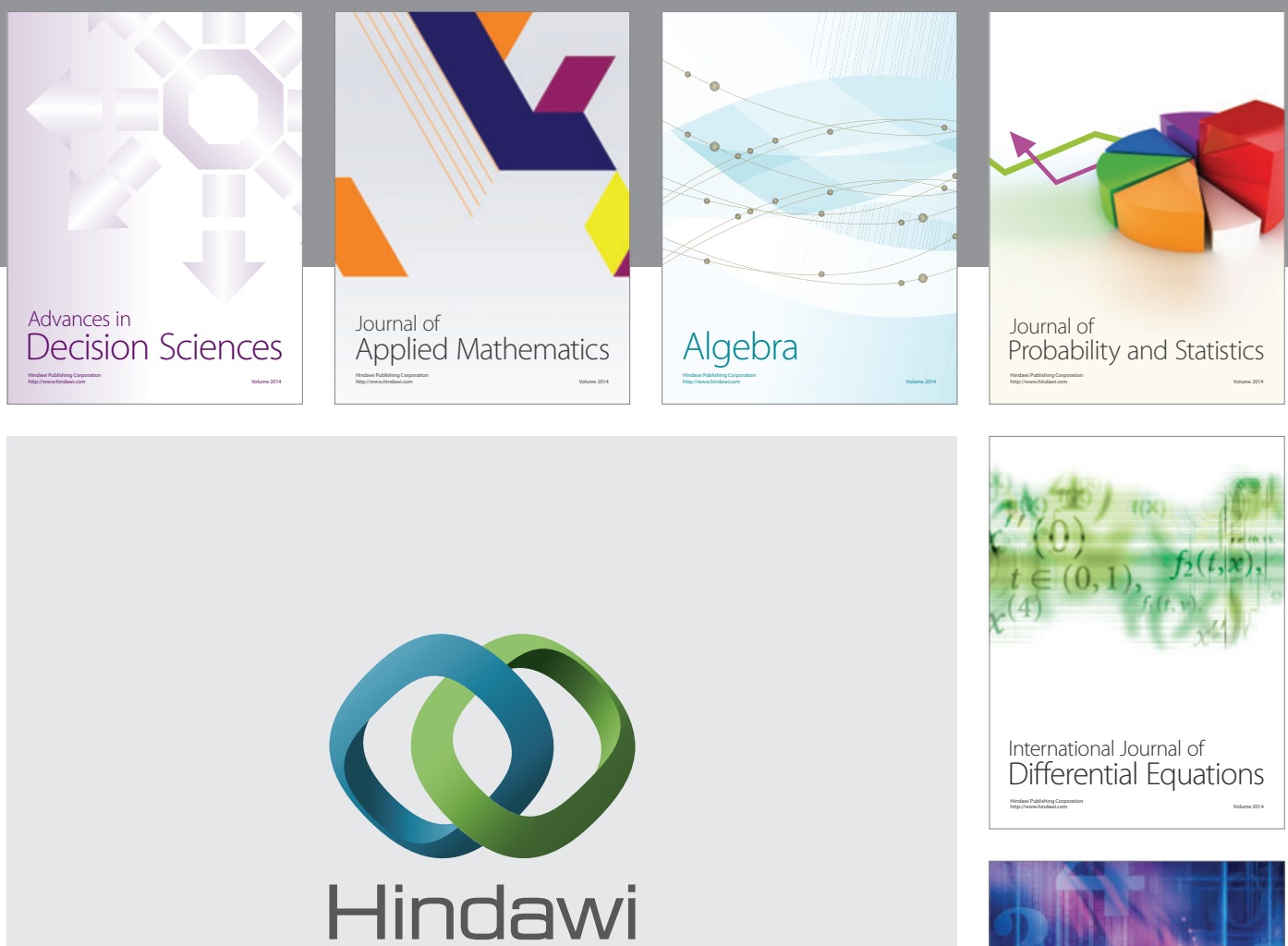

Submit your manuscripts at http://www.hindawi.com
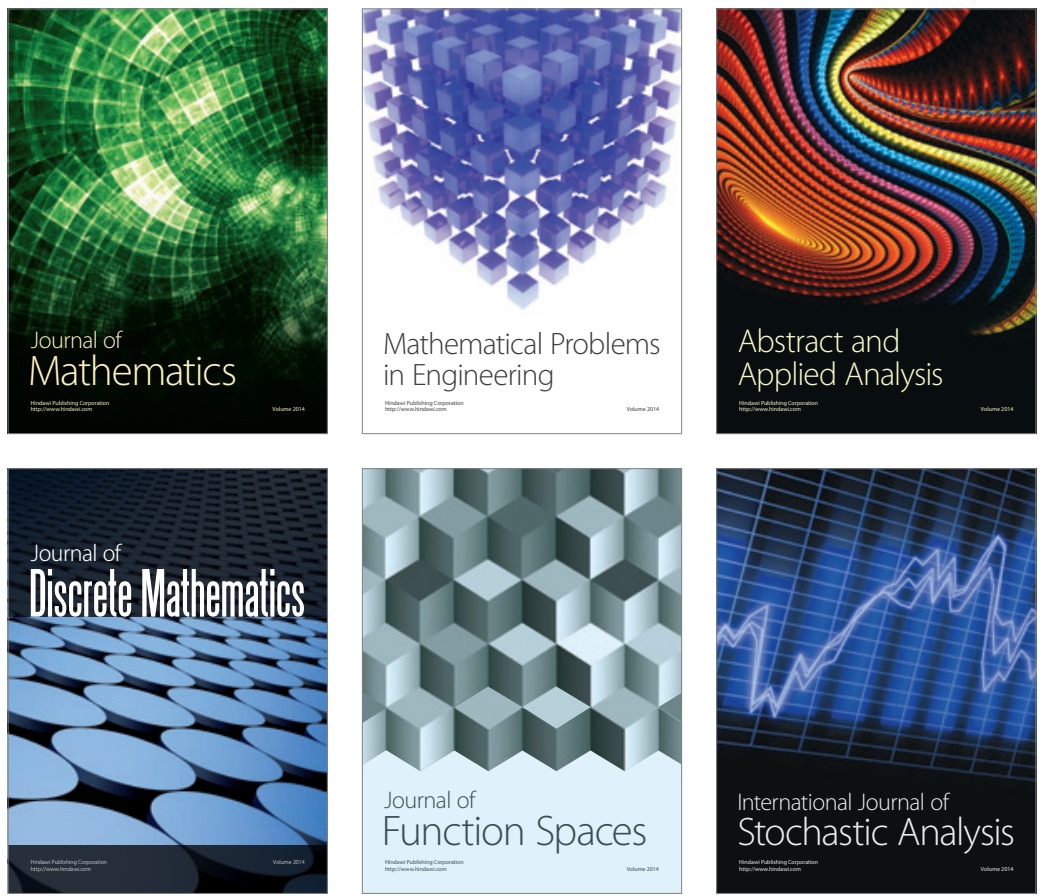

Journal of

Function Spaces

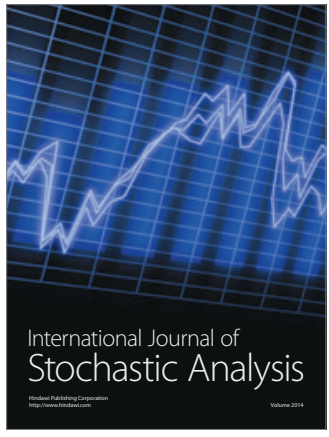

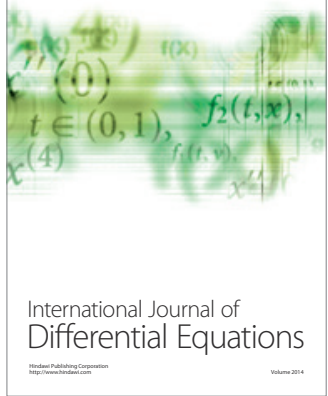
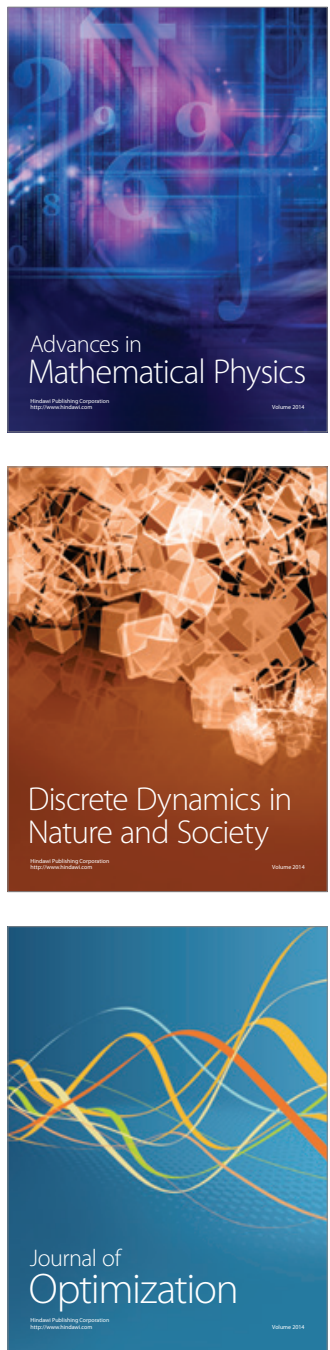